\title{
Time-dependent matrix product ansatz for interacting reversible dynamics
}

\author{
Katja Klobas, Marko Medenjak, Tomaž Prosen, Matthieu \\ Vanicat \\ Department of Physics, Faculty of Mathematics and Physics, University of Ljubljana, \\ Ljubljana, Slovenia
}

\begin{abstract}
We present an explicit time-dependent matrix product ansatz (tMPA) which describes the time-evolution of any local observable in an interacting and deterministic lattice gas, specifically for the rule 54 reversible cellular automaton of [Bobenko et al. Commun. Math. Phys. 158, 127 (1993)]. Our construction is based on an explicit solution of real-space real-time inverse scattering problem. We consider two applications of this tMPA. Firstly, we provide the first exact and explicit computation of the dynamic structure factor in an interacting deterministic model, and secondly, we solve the extremal case of the inhomogeneous quench problem, where a semi-infinite lattice in the maximum entropy state is joined with an empty semi-infinite lattice. Both of these exact results rigorously demonstrate a coexistence of ballistic and diffusive transport behaviour in the model, as expected for normal fluids.
\end{abstract}

\section{Introduction}

Understanding rigorously how the macroscopic hydrodynamic behaviour of interacting particle systems emerges from the microscopic description is one of the major quests of nonequilibrium statistical mechanics [1]. This problem is particularly hard in systems, where the microscopic equations of motion are reversible and no external sources of noise or dissipation are built into the model. The standard route of deriving the macroscopic description for the interacting systems with hyperbolic (i.e. 'chaotic') microscopic dynamics, say for elastically colliding hard-spheres, goes via the justification of the Boltzmann equation, which is possible in the Boltzmann-Grad limit [2]. Despite this limitation, the hydrodynamic approach has been recently heuristically demonstrated even in quantum integrable systems $[3[6]$ where any possible mechanism of chaos is absent.

In this paper we discuss an interacting deterministic many-body classical system in a single spatial dimension, for which we can explicitly compute the complete dynamics of all local observables. This is a reversible cellular automaton given by the rule 54 (RCA 54) of Bobenko et al [7], also related to a model coded as ERCA 250R introduced by Takesue 8 . The model is a two state locally interacting deterministic lattice system that describes the dynamics of classical solitons with nontrivial pairwise scattering. 
Recently non-equilibrium steady state of the system coupled to stochastic reservoirs has been found [9, 10], as well as some of its decay modes [11]. These results suggest that the model can be indeed considered as an integrable Hamiltonian system although no Lax zero curvature formulation of the equations of motion has been found to date. The model has as well been studied in the quantum context, since it can also be interpreted as a quantum cellular automaton describing spreading of time dependent local operators [12].

The key new concept in our work is an exact and explicit time dependent matrix product anastz (tMPA) representation of time-evolution of local observables. The dimension of the auxiliary space which supports the matrix representation is formally infinite, but in time $t$ it only explores a polynomial, in fact $\mathcal{O}\left(t^{2}\right)$, dimensional subspace, which implies efficient computation of dynamics. As an application of our technique we provide explicit and large time/space asymptotic results of the dynamic structure factor (i.e. spatio-temporal density-density correlation function in the maximum entropy equilibrium state) as well as an explicit solution of the inhomogeneous quench problem of joining a maximum entropy semi-infinite lattice with an empty semi-infinite lattice. Both explicit solutions demonstrate a coexistence of ballistic (convective) and diffusive (conductive) transport, which is typically to be expected in normal gasses or liquids.

The paper is structured as follows: In section 2 we describe the dynamics of the RCA 54. In section 3 we present the main result; the time propagation of the local observables is expressed as a tMPA, which is explicitly derived by solving a real-time real-space inverse scattering problem. Despite the infinite dimensionality of the tMPA, we are able to obtain the solution of two physically interesting problems by exploiting the structure of the matrices (as explained in Appendix A). In section 4 we solve the inhomogeneous quench problem, where the left-hand side of the system is prepared in the maximally mixed state, and the right-hand side is completely vacant. The second problem we address is the analytical calculation of the dynamic structure factor, presented in section 5 .

\section{The model}

\subsection{Definition of the dynamics}

The model is defined on the infinite chain $\mathbb{Z}$, and each site of the chain can be either occupied or empty. The configuration of the system at time $t$ is given by a string of binary digits $\underline{s}^{t}=\left(\ldots, s_{-1}^{t}, s_{0}^{t}, s_{1}^{t}, s_{2}^{t}, \ldots\right) \in\{0,1\}^{\mathbb{Z}}$, where $s_{x}^{t}=0$, if the site $x$ is empty at time $t$ and $s_{x}^{t}=1$, if it is occupied. The states are put on a saw shaped lattice, and the dynamics is provided by the staggered reversible deterministic discrete space-time mapping

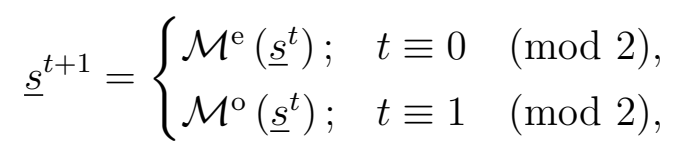



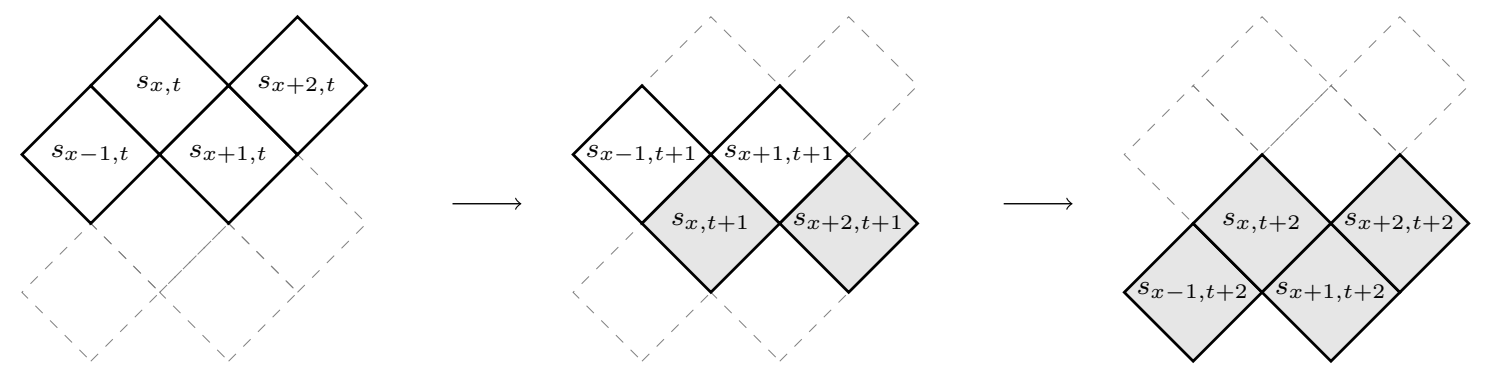

Figure 1. Schematic representation of the time evolution of a section of the lattice. In the first time-step, only the sites $x$ and $x+2$ are updated, while the states on the sites $x-1$ and $x+1$ remain unchanged. In the second time step, the sites $x \pm 1$ are updated, and $x, x+2$ do not change.

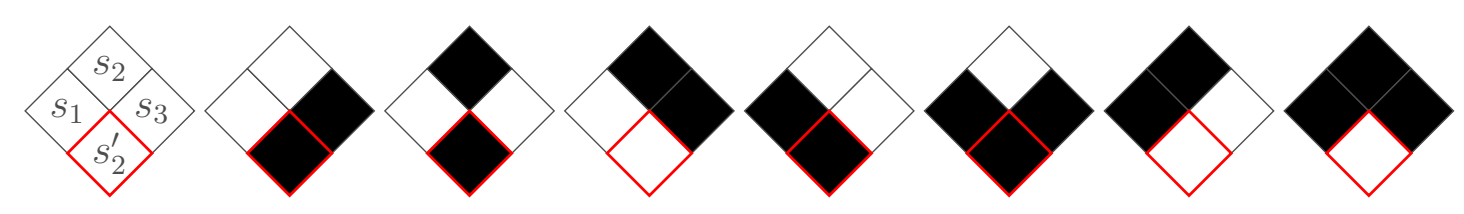

Figure 2. In the figure, the RCA 54 is presented diagrammatically. The updated value $s_{2}^{\prime}$, i.e. the square with a red-border, depends on the values $s_{1}, s_{2}, s_{3}$ (the top three squares) and is given by the local map $s_{2}^{\prime}=\chi\left(s_{1}, s_{2}, s_{3}\right)$ as defined in 2.3 .

where $\mathcal{M}^{\mathrm{e}}: \underline{s} \mapsto \underline{s}^{\prime}$ and $\mathcal{M}^{\mathrm{o}}: \underline{s} \mapsto \underline{s}^{\prime \prime}$ are maps from $\{0,1\}^{\mathbb{Z}}$ to $\{0,1\}^{\mathbb{Z}}$, defined by the local three-site updates

$s_{x}^{\prime}=\left\{\begin{array}{ll}\chi\left(s_{x-1}, s_{x}, s_{x+1}\right) ; & x \equiv 0(\bmod 2), \\ s_{x} & x \equiv 1(\bmod 2),\end{array} \quad s_{x}^{\prime \prime}= \begin{cases}s_{x} ; & x \equiv 0(\bmod 2) \\ \chi\left(s_{x-1}, s_{x}, s_{x+1}\right) ; & x \equiv 1(\bmod 2)\end{cases}\right.$

The schematic representation of the time evolution is presented in Figure 1. The RCA 54 is described by the binary function $\chi$,

$$
\chi\left(s_{1}, s_{2}, s_{3}\right)=s_{1}+s_{2}+s_{3}+s_{1} s_{3} \quad(\bmod 2) .
$$

The rules (2.3) are diagrammatically expressed in Figure 2, The complete time evolution is obtained by alternately applying the maps $\mathcal{M}^{\mathrm{e}}$ and $\mathcal{M}^{\mathrm{o}}$. Note that $\mathcal{M}^{\mathrm{e}}$ and $\mathcal{M}^{\mathrm{o}}$ encode exactly the same rules shifted by a single lattice site. The dynamics induced by the mapping 2.1 is time-reversible since the relation

$$
\chi\left(s_{1}, \chi\left(s_{1}, s_{2}, s_{3}\right), s_{3}\right)=s_{2},
$$

is satisfied for all $s_{1}, s_{2}, s_{3} \in\{0,1\}$.

Alternatively, the time propagation (2.1) can be represented by the following prescription

$$
s_{x}^{t+1}=\left\{\begin{array}{lll}
\chi\left(s_{x-1}^{t}, s_{x}^{t}, s_{x+1}^{t}\right) ; & x+t \equiv 0 & (\bmod 2), \\
s_{x}^{t} ; & x+t \equiv 1 & (\bmod 2) .
\end{array}\right.
$$

The physical interpretation of the dynamics induced by the RCA 54 is rather simple. Occupied sites can be interpreted as the solitons moving with a constant velocity \pm 1 , or two scattering solitons, depending on the states of the neighboring two sites. After 


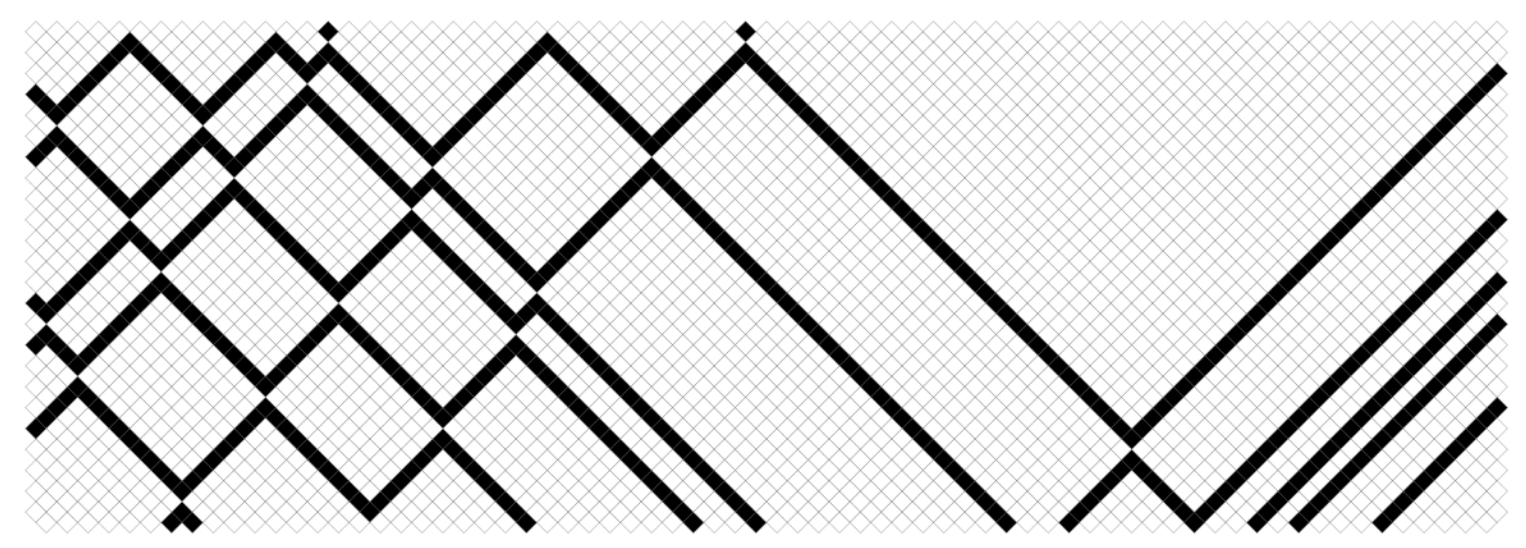

Figure 3. Time evolution of a random initial configuration. Particles move with velocity 1 and scatter pairwise by obtaining a time shift.

the scattering both solitons are time-delayed for a single time-step, see Figure 3. The solitons with velocity \pm 1 will be called the left- or right-movers, respectively.

In the following paragraphs we introduce the necessary prerequisites in order to explicitly study statistical mechanics of the model.

\subsection{Algebra of local observables}

In order to express and efficiently compute the expectation values of physical quantities, we introduce a commutative quasi-local $C^{*}$ algebra $\mathcal{A}$ of observables, $\ddagger$ or functions over $\{0,1\}^{\mathbb{Z}}$. Any local subalgebra $\mathcal{A}_{x} \subset \mathcal{A}$ pertaining to the site $x \in \mathbb{Z}$ is spanned by the local basis $[\alpha]_{x}, \alpha \in\{0,1\}$, defined by the following relation and the multiplication rule

$$
[\alpha]_{x}(\underline{s})=\delta_{\alpha, s_{x}}, \quad\left([\alpha]_{x}[\beta]_{y}\right)(\underline{s})=[\alpha]_{x}(\underline{s})[\beta]_{y}(\underline{s}),
$$

where $\underline{s}=\left(\ldots, s_{-1}, s_{0}, s_{1}, \ldots\right)$ is an arbitrary configuration of occupied and empty sites. A product of local observables on $r$ consecutive sites centered around $x$ is denoted by

$$
\left[\alpha_{1} \alpha_{2} \ldots \alpha_{r}\right]_{x} \equiv\left[\alpha_{1}\right]_{x-\left\lfloor\frac{r}{2}\right\rfloor}\left[\alpha_{2}\right]_{x-\left\lfloor\frac{r}{2}\right\rfloor+1} \cdots\left[\alpha_{r}\right]_{x+\left\lfloor\frac{r-1}{2}\right\rfloor}
$$

and spans a complete function algebra over a finite sublatice $\mathcal{A}_{[y, z]}=\bigotimes_{x=y}^{z} \mathcal{A}_{x}$, with $y=x-\left\lfloor\frac{r}{2}\right\rfloor, z=x+\left\lfloor\frac{r-1}{2}\right\rfloor$. For conciseness we sometimes omit the subscript, in which case it is assumed to be 0 ,

$$
\left[\alpha_{1} \alpha_{2} \ldots \alpha_{r}\right] \equiv\left[\alpha_{1} \alpha_{2} \ldots \alpha_{r}\right]_{0} .
$$

The quasilocal algebra $\mathcal{A}$ can then be understood as an appropriate norm-closure of an inclusive sequence $\mathcal{A}_{[-z, z]} \subset \mathcal{A}_{[-z-1, z+1]} \subset \mathcal{A}_{[-z-2, z+2]} \cdots$.

Let us introduce an identity observable $\mathbb{1}_{x}=[0]_{x}+[1]_{x}$, a unit element in $\mathcal{A}_{x}$. Any observable $a \in \mathcal{A}$ is preserved under multiplication by $\mathbb{1}_{x}$ (which in fact represent the $\ddagger \mathcal{A}$ can be considered as a subalgebra (of diagonal operators, i.e. those jointly commuting with $z$ components of all local spin operators) of the quasi-local spin $1 / 2$ UHF algebra [13. 
same (unit) element in $\mathcal{A}, \mathbb{1}_{x} \equiv \mathbb{1}$ )

$$
a \mathbb{1}_{x}=\mathbb{1}_{x} a=a,
$$

therefore we can extend the support of any local observable multiplying by any number of identities at the edges, e.g.

$$
\begin{aligned}
{\left[\alpha_{1} \alpha_{2} \ldots \alpha_{r}\right]_{x} } & \equiv \mathbb{1}_{x-\left\lfloor\frac{r+2}{2}\right\rfloor} \cdot\left[\alpha_{1} \alpha_{2} \ldots \alpha_{r}\right]_{x} \cdot \mathbb{1}_{x+\left\lfloor\frac{r+1}{2}\right\rfloor} \equiv \\
& \equiv\left[0 \alpha_{1} \alpha_{2} \ldots \alpha_{r} 0\right]_{x}+\left[0 \alpha_{1} \alpha_{2} \ldots \alpha_{r} 1\right]_{x}+\left[1 \alpha_{1} \alpha_{2} \ldots \alpha_{r} 0\right]_{x}+\left[1 \alpha_{1} \alpha_{2} \ldots \alpha_{r} 1\right]_{x}
\end{aligned}
$$

\subsection{Expectation values and states}

For discussing statistical mechanics of the RCA 54, we introduce the notion of separable states, for which $\left\{s_{x}\right\}_{x \in \mathbb{Z}}$ are Bernoulli random variables, corresponding to independent probability distributions $p_{x}: s_{x} \rightarrow[0,1]$ for all $x \in \mathbb{Z}$. The expectation values of a local observable $\left[\alpha_{1} \alpha_{2} \ldots \alpha_{r}\right]_{x}$ in a separable state $p, p \equiv$ $\left(\ldots,\left\{p_{-1}(0), p_{-1}(1)\right\},\left\{p_{0}(0), p_{0}(1)\right\},\left\{p_{1}(0), p_{1}(1)\right\} \ldots\right)$, is given by the prescription

$$
\left\langle\left[\alpha_{1} \alpha_{2} \ldots \alpha_{r}\right]_{x}\right\rangle_{p}=p_{x-\left\lfloor\frac{r}{2}\right\rfloor}\left(\alpha_{1}\right) \cdot p_{x-\left\lfloor\frac{r}{2}\right\rfloor+1}\left(\alpha_{2}\right) \cdots p_{x+\left\lfloor\frac{r-1}{2}\right\rfloor}\left(\alpha_{r}\right) .
$$

For example, in the following sections we will consider two particular initial states:

(i) A maximum entropy state in section 5 defined by

$$
p_{x}(0)=p_{x}(1)=1 / 2, \quad \forall x \in \mathbb{Z} .
$$

(ii) An inhomogeneous initial state in section 4 defined by

$$
\begin{cases}p_{x}(0)=p_{x}(1)=1 / 2, & \text { for } x \leq 0 \\ p_{x}(0)=1, \quad p_{x}(1)=0 . & \text { for } x>0\end{cases}
$$

One could as well consider expectation values of local observables in a more general setup, where the states are not necessarily separable but satisfy a general clustering property, i.e. the distribution of variable $s_{x}$ may depend on the values $\left\{s_{y}\right\}$ for $y$ sufficiently close to $x$, i.e., for $|x-y| \rightarrow \infty,\left\langle[\alpha]_{x}[\beta]_{y}\right\rangle_{p} \rightarrow\left\langle[\alpha]_{x}\right\rangle_{p}\left\langle[\alpha]_{y}\right\rangle_{p}$.

\subsection{Time evolution of local observables}

The dynamics on the configuration space induces the time-evolution of local observables, which corresponds to the time automorphism of the quasi-local algebra $\mathcal{A}$. For any observable $a \in \mathcal{A}$, we define its time evolved version $a^{t} \in \mathcal{A}$ as

$$
a^{t}\left(\underline{s}^{0}\right)=a\left(\underline{s}^{t}\right) .
$$

Explicitly, a local time automorphism $U_{x}$ is defined by its action on 3-site local observables by

$$
U_{x}[\alpha \beta \gamma]_{y}= \begin{cases}{[\alpha \chi(\alpha, \beta, \gamma) \gamma]_{y} ;} & x=y \\ {[\alpha \beta \gamma]_{y} ;} & |x-y| \geq 2\end{cases}
$$


and is extended to any quasilocal observable $a \in \mathcal{A}$ by linearity and continuity. The complete time evolution of the observables

$$
a^{t+1}=U(t) a^{t}
$$

is then given by composed linear automorphism $U(t)$, which depends on the parity of time $t$

$$
U(t)=\left\{\begin{array}{lll}
\prod_{x \in 2 \mathbb{Z}} U_{x} ; & t \equiv 0 & (\bmod 2) \\
\prod_{x \in 2 \mathbb{Z}+1} U_{x} ; & t \equiv 1 & (\bmod 2)
\end{array}\right.
$$

\section{Construction of time-dependent matrix product ansatz}

In this section we present our main result, which is the derivation of the complete dynamics of local observables in terms of tMPA. Using the homomorphism property of the time automorphism

$$
\left[\alpha_{1} \alpha_{2} \ldots \alpha_{r}\right]_{x}^{t} \equiv\left[\alpha_{1}\right]_{x-\left\lfloor\frac{r}{2}\right\rfloor}^{t}\left[\alpha_{2}\right]_{x-\left\lfloor\frac{r}{2}\right\rfloor+1}^{t} \cdots\left[\alpha_{r}\right]_{x+\left\lfloor\frac{r-1}{2}\right\rfloor}^{t},
$$

it is possible to construct the tMPA of any local observable by tensor multiplying tMPAs for the single site observables. Additionally, the stationarity of the identity observable $\mathbb{1}_{x}^{t} \equiv \mathbb{1}$ implies that the time evolution of the observable $[0]_{x}$ can be expressed in terms of the observable $[1]_{x}$ as

$$
[0]_{x}^{t}=\mathbb{1}-[1]_{x}^{t}
$$

To construct the tMPA of an arbitrary local observable it is thus sufficient to consider the time propagation of $[1]_{x}$. The problem can be further reduced by noting that the time propagation of the shifted observable can be obtained by appropriately shifting the time propagated observable centered at the origin

$$
[1]_{x}^{t}=\left\{\begin{array}{lll}
\eta_{x}\left([1]^{t}\right) ; & x \equiv 0 & (\bmod 2), \\
\eta_{x}\left([1]^{t-1}\right) ; & x \equiv 1 & (\bmod 2) .
\end{array}\right.
$$

Here $\eta_{x}$ is the lattice shift automorphism of $\mathcal{A}$ defined as $\eta_{x}\left([\underline{s}]_{y}\right) \equiv[\underline{s}]_{x+y}$.

Theorem 1. The time evolution of the local observable [1] reads

$$
[1]^{t}=\sum_{s_{-t}, \ldots, s_{t} \in\{0,1\}} c_{s_{-t}, \ldots, s_{t}}(t)\left[s_{-t} s_{-t+1} \cdots s_{t}\right]
$$

where the amplitudes $c_{s_{-t}, \ldots, s_{t}}(t)$ can be represented in terms of the $t M P A$

$$
\begin{aligned}
c_{s_{-t}, \ldots s_{t}}(t) & =\left\langle l(t)\left|V_{s_{-t}} W_{s_{-t+1}} V_{s_{-t+2}} \cdots W_{s_{t-1}} V_{s_{t}}\right| r\right\rangle \\
& +\left\langle l^{\prime}\left|V_{s_{-t}}^{\prime} W_{s_{-t+1}}^{\prime} V_{s_{-t+2}}^{\prime} \cdots W_{s_{t-1}}^{\prime} V_{s_{t}}^{\prime}\right| r^{\prime}(t)\right\rangle .
\end{aligned}
$$

$V_{s}, W_{s}, V_{s}^{\prime}, W_{s}^{\prime} \in \operatorname{End}(\mathcal{V}), s \in\{0,1\}$, are linear operators over an infinite dimensional auxiliary Hilbert space $\mathcal{V}=\operatorname{lsp}\left\{|c, w, n, a\rangle ; c, w \in \mathbb{N}_{0}, n \in\{0,1,2\}, a \in\{0,1\}\right\}$, and can be explicitly expressed in terms of ladder operators

$$
\begin{array}{ll}
\mathbf{c}^{+}=\sum_{c, w, n, a}|c+1, w, n, a\rangle\langle c, w, n, a|, & \mathbf{c}^{-}=\left(\mathbf{c}^{+}\right)^{T}, \\
\mathbf{w}^{+}=\sum_{c, w, n, a}|c, w+1, n, a\rangle\langle c, w, n, a|, & \mathbf{w}^{-}=\left(\mathbf{w}^{+}\right)^{T},
\end{array}
$$


and projectors

$$
\begin{aligned}
& \mathbf{e}_{c_{2} w_{2} n_{2} a_{2}, c_{1} w_{1} n_{1} a_{1}}=\left|c_{2}, w_{2}, n_{2}, a_{2}\right\rangle\left\langle c_{1}, w_{1}, n_{1}, a_{1}\right|, \\
& \mathbf{e}_{n_{2} a_{2}, n_{1} a_{1}}=\sum_{c, w}\left|c, w, n_{2}, a_{2}\right\rangle\left\langle c, w, n_{1}, a_{1}\right|
\end{aligned}
$$

as

$$
\begin{aligned}
V_{0} & =\mathbf{e}_{00,00}+\mathbf{e}_{10,00}+\mathbf{e}_{20,00}+\mathbf{c}^{+} \mathbf{e}_{10,01}+\mathbf{e}_{01,01}+\mathbf{c}^{+} \mathbf{w}^{+} \mathbf{e}_{11,01}+\mathbf{e}_{21,01}+ \\
& +\mathbf{e}_{0001,0001}+\mathbf{e}_{0011,0001}+\mathbf{e}_{0021,0001} \\
V_{1} & =\mathbf{e}_{00,10}+\mathbf{e}_{10,20}+\mathbf{e}_{20,20}+\mathbf{e}_{00,11}+\mathbf{e}_{10,21}+\mathbf{e}_{20,21}+\mathbf{e}_{01,11}+\mathbf{w}^{+} \mathbf{e}_{11,21}+\mathbf{w}^{+} \mathbf{e}_{21,21}+ \\
& +\mathbf{e}_{0001,0011}+\mathbf{e}_{0011,0021}+\mathbf{e}_{0021,0021} \\
W_{0} & =\mathbf{c}^{-} \mathbf{w}^{+}\left(\mathbf{e}_{00,00}+\mathbf{e}_{10,00}+\mathbf{e}_{20,00}\right)+\mathbf{w}^{+} \mathbf{e}_{10,01}+\mathbf{w}^{+} \mathbf{e}_{01,01}+\mathbf{c}^{+}\left(\mathbf{w}^{+}\right)^{2} \mathbf{e}_{11,01}+ \\
& +\mathbf{w}^{+} \mathbf{e}_{21,01}+\mathbf{e}_{1111,0001}+\mathbf{e}_{0001,0001}+\mathbf{e}_{0011,0001}+\mathbf{e}_{0021,0001} \\
W_{1} & =\mathbf{c}^{-} \mathbf{w}^{+}\left(\mathbf{e}_{00,10}+\mathbf{e}_{10,20}+\mathbf{e}_{20,20}\right)+\mathbf{w}^{+} \mathbf{e}_{01,11}+\mathbf{c}^{+} \mathbf{w}^{+} \mathbf{e}_{11,21}+\mathbf{c}^{+} \mathbf{w}^{+} \mathbf{e}_{21,21}+ \\
& +\mathbf{e}_{0001,0011}+\mathbf{e}_{0011,0021}+\mathbf{e}_{0021,0021},
\end{aligned}
$$

and

$$
\begin{aligned}
& V_{0}^{\prime}=V_{0}^{T}-\left(\mathbf{e}_{0001,1111}+\mathbf{e}_{0101,1211}+\mathbf{e}_{0101,1110}\right), \\
& V_{1}^{\prime}=V_{1}^{T}, \\
& W_{0}^{\prime}=W_{0}^{T}-\left(\mathbf{e}_{0001,1111}+\mathbf{e}_{0000,1211}\right), \\
& W_{1}^{\prime}=W_{1}^{T}-\left(\mathbf{e}_{0021,1111}+\mathbf{e}_{0021,1121}+\mathbf{e}_{0121,1211}+\mathbf{e}_{0121,1221}\right) .
\end{aligned}
$$

The time-dependent auxiliary space boundary vectors take the following form

$$
\begin{aligned}
& \langle l(t)|=\langle 0, t, 0,0|, \\
& |r\rangle=|0,0,0,0\rangle+|0,0,0,1\rangle+|0,0,0,2\rangle, \\
& \left\langle l^{\prime}\right|=\langle 0,0,0,1|+\langle 0,0,1,1|+\langle 0,0,2,1|+\langle 0,1,0,1|+\langle 0,1,2,1|, \\
& \left|r^{\prime}(t)\right\rangle=|0, t+1,0,0\rangle .
\end{aligned}
$$

Remark. Before dwelling into the full-blown proof, let us first elucidate the main idea leading to a compact description of the dynamics and its properties.

First of all note that the reduction of the dynamics of the ultra-local observable to the sub-lattice of the size $2 t+1$ around the origin is a consequence of the locality of the time evolution operator $U$, which maps the subalgebra of local observables $\mathcal{A}_{[-t, t]}$ to the subalgebra of local observables with the increased support $\mathcal{A}_{[-t-1, t+1]}$, and can thus be equivalently expressed in terms of the reduced propagator

$$
U_{[-t-1, t+1]} \equiv U_{-t} U_{-t+2} \cdots U_{t}
$$

To be more concise, the dynamics of the local observable $\left[s_{-t} \cdots s_{t}\right]$ is completely determined by the following prescription

$$
\begin{aligned}
& U(t)\left[s_{-t} \cdots s_{t}\right] \equiv \\
& U_{[-t-1, t+1]}\left(\left[0 s_{-t} \cdots s_{t} 0\right]+\left[0 s_{-t} \cdots s_{t} 1\right]+\left[1 s_{-t} \cdots s_{t} 0\right]+\left[1 s_{-t} \cdots s_{t} 1\right]\right) .
\end{aligned}
$$

The fact that we have a deterministic dynamics has two immediate consequences. Firstly, the coefficients $c_{s_{-t}, \ldots s_{t}}(t)$ can only be 0, corresponding to the inaccessible 


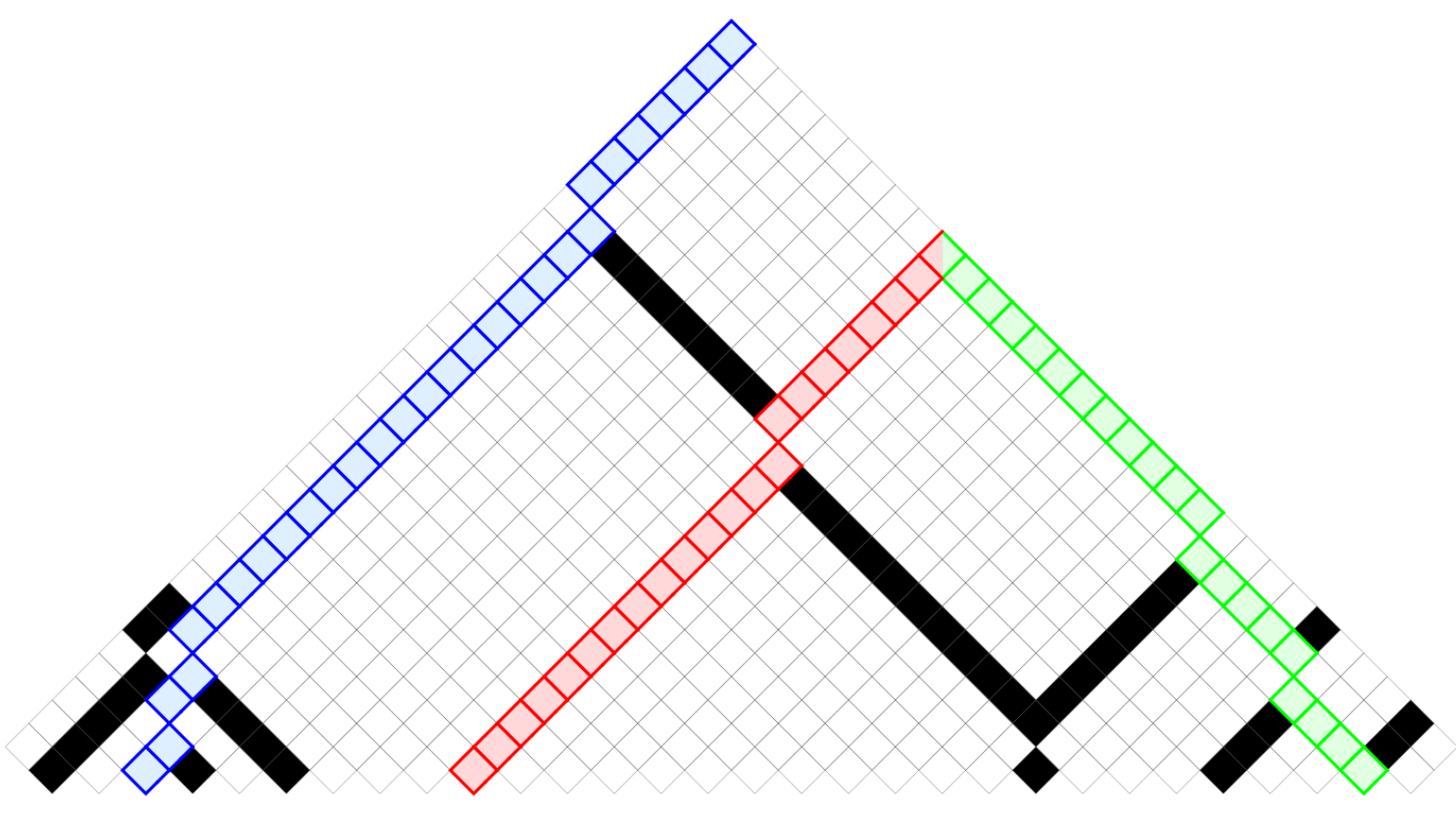

Figure 4. An example of an allowed configuration (the bottom-most saw). The red, blue and green-colored sites denote three distinct solitons. The blue soliton goes through the site $(0,0)$, while the red and green solitons originate outside of the lightcone. Alternatively, we can think of this as solitons that start at the bottom and propagate in the negative time. The blue soliton goes through the top site, while the red and green one escape the light-cone and cannot reach the origin.

configurations, or 1, corresponding to the accessible configurations. Secondly, the number of accessible configurations at time $t$ is $4^{t}$ which is half of all possible distinct configurations $2^{2 t+1}$.

The construction of the tMPA relies on an explicit identification of all accessible configurations. The initial configuration is [1], describing all possible states with at least one soliton traversing through the origin at time $t=0$. At time $t$, the soliton originating from the center resides between the lattice sites $x=-t$ and $x=t$, i.e. the section of the lattice referred to as a light-cone. The exact position of the soliton is determined by the number of scatterings $c$. An example is shown in the Figure 4 .

The configuration at time $t$ contains the complete information about the particle content and the scatterings, implying that we can propagate any configuration backwards in time in order to determine whether one of the solitons originated from the central position. The tMPA is constructed so that it traces every particle backwards in time, by counting the number of scatterings, and determines whether a given particle ends up at the origin. If this is the case, tMPA coefficient yields 1, otherwise the contribution vanishes.

Proof. The proof of theorem 1 consists of two parts. In the first part we derive the tMPA for the states in which the soliton emerging from the center is a left mover, and in the second part for the central right movers. 
tMPA for the left mover emerging from the center

Let us consider a configuration $\left[s_{-t} \cdots s_{t}\right] \in\{0,1\}^{2 t+1}$. Using the tMPA we scan a given configuration site by site, starting from the left edge of the light-cone and moving towards the right edge. Whenever we encounter a left mover, which we dub the probe, we count the number of solitons on its right in order to determine, whether it originated from the center. To encode the soliton counting procedure, we introduce four auxiliary degrees of freedom, $|c, w, n, a\rangle$ :

(i) The activation bit, $a \in\{0,1\}$, tells us whether we are on the left or the right side of the probe. If the activation bit is turned off, i.e. $a=0$, the state splits into two parts whenever we encounter a left mover. The first part corresponds to the value $a=0$, describing the situation in which the left mover is not a probe, while the second part represents the opposite case, with $a=1$. In any other situation the activation bit remains unchanged.

(ii) The collision counter $c \in \mathbb{N}_{0}$, represents the number of scatterings that the probe has to undergo in order to reach the origin in the inverse scattering procedure, and at the same time it distinguishes between the different probes of the same state. While $a=0$, the collision counter increases by 1 every two sites. If $a=1$, the collision counter decreases by 1 whenever a left moving soliton that scattered with the probe is encountered. If at the right edge of the light cone the collision counter is zero, the probe passed through the origin.

(iii) The scattering width $w \in \mathbb{N}_{0}$, keeps track of the scatterings of the right movers after the probe. At the left edge the width is equal to time $t$, and after every two sites it decreases by 1 . Additionally, the width changes as $w \rightarrow w-1$, whenever a left mover on the right side of the probe is encountered. If the width drops to 0 , the right movers that we meet did not scatter with the probe.

(iv) The occupation counter $n \in\{0,1,2\}$ provides additional information about the particle content needed to appropriately change $w$ and $c$. Explicitly, $n=0$ if the current site is empty, $n=1$ if the site is full and the left neighbor is empty, and $n=2$ if the site and the left neighbor are both occupied.

In the initial state the collision counter $c$ is 0 , and the width $w$ is set to the number of time-slices $t$,

$$
\langle l(t)|=\langle 0, t, 0,0| .
$$

The right boundary vector has nonzero overlap with vectors that correspond to a probe that passed through the origin at time $t=0$, i.e. $c=w=0$ and $a=1$, while the occupation number can be arbitrary,

$$
|r\rangle=|0,0,0,1\rangle+|0,0,1,1\rangle+|0,0,2,1\rangle .
$$


Before constructing the matrix elements, let us first introduce the projector to the subspace with $a=0$,

$$
P_{0}=\sum_{c, w, n}|c, w, n, 0\rangle\langle c, w, n, 0|=P_{0}^{T}=P_{0}^{2},
$$

and consider three different regimes.

Left side of the probe Before choosing the probe, the width and the required number of scatterings have to be adjusted, therefore the left action of the restricted matrices $W_{s} P_{0}, V_{s} P_{0}$ corresponds to

$$
\begin{aligned}
& \langle c, w, n, 0| V_{s} P_{0}=\langle c, w, s \cdot \min \{n+1,2\}, 0|, \\
& \langle c, w, n, 0| W_{s} P_{0}=\langle c+1, w-1, s \cdot \min \{n+1,2\}, 0| .
\end{aligned}
$$

Choosing the probe Whenever a left moving soliton is encountered, an additional vector with $a=1$ is created. There are 4 such configurations. The two simpler ones correspond to a soliton that appears on the right diagonal,

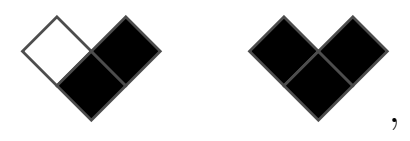

which implies

$$
\langle c, w, 1,0| V_{1}\left(1-P_{0}\right)=\langle c, w, 2,0| V_{1}\left(1-P_{0}\right)=\langle c, w, 2,1| .
$$

The other two configurations correspond to encountering a soliton while it is scattering,
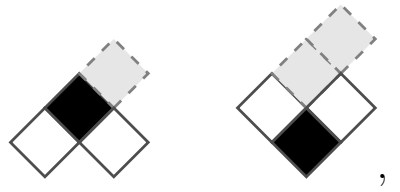

where the grey colored squares represent the soliton's estimated path in absence of any additional encounters. The appropriate matrix elements are the following,

$$
\begin{aligned}
& \langle c, w, 1,0| W_{0}\left(1-P_{0}\right)=\langle c, w-1,0,1|, \\
& \langle c, w, 1,0| V_{0}\left(1-P_{0}\right)=\langle c-1, w, 0,1| .
\end{aligned}
$$

Right side of the probe Let us assume that $w>0$. Once a vector with $a=1$ is produced, the collision counter has to be decreased whenever a right mover is encountered, while the width $w$ decreases every two sites and additionally whenever a left mover is met. Explicitly, there are two possible configurations of a right mover appearing,

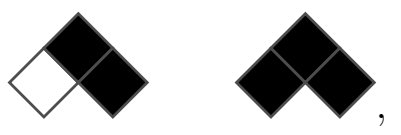

which are described by the following matrix elements,

$$
\langle c, w, 1,1| W_{1}=\langle c, w, 2,1| W_{1}=\langle c-1, w-1,2,1| .
$$


The two configurations from 3.17 correspond to a process of encountering a left moving soliton, which is described by the following two matrix elements

$$
\langle c, w, 1,1| V_{1}=\langle c, w, 2,1| V_{1}=\langle c, w-1,2,1| .
$$

If the scattering solitons are encountered (eq. (3.19)), we have to decrease the width and the collision counter at the same time, which amounts to the following,

$$
\begin{aligned}
& \langle c, w, 1,1| W_{0}= \begin{cases}0 ; & w=1 \text { and } c \neq 1, \\
\langle 0,0,0,1| ; & w=c=1 \\
\langle c-1, w-2,0,1| ; & w \geq 2 .\end{cases} \\
& \langle c, w, 1,1| V_{0}=\langle c-1, w-1,0,1| .
\end{aligned}
$$

In all the remaining cases,
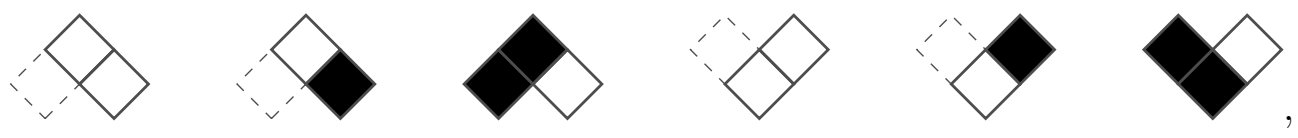

there are no additional decreases of $w$ and $c$, therefore

$$
\begin{array}{ll}
\langle c, w, 0,1| W_{0}=\langle c, w-1,0,1|, & \langle c, w, 0,1| W_{1}=\langle c, w-1,1,1|, \\
\langle c, w, 2,1| W_{0}=\langle c, w-1,0,1|, & \langle c, w, 0,1| V_{0}=\langle c, w, 0,1|, \\
\langle c, w, 0,1| V_{1}=\langle c, w, 1,1|, & \langle c, w, 2,1| V_{0}=\langle c, w, 0,1| .
\end{array}
$$

The right movers that are encountered after the width $w$ drops to 0 did not scatter with the probe, therefore $c$ should not decrease anymore. The probe reached the origin only if the value of the collision counter $c$ is 0 on the right side of the light-cone, inducing the following matrix elements

$$
\langle c, 0, n, 1| W_{s}=\langle c, 0, n, 1| V_{s}= \begin{cases}\langle 0,0, s \cdot \max \{2, n+1\}, 1| ; & c=0 \\ 0 ; & c>0\end{cases}
$$

which completes the construction of the tMPA for the left movers (3.8).

\section{tMPA for the central right movers}

The tMPA of the right movers can be derived in a similar fashion, by reversing the direction of all solitons. This corresponds to exchanging the roles of the left and the right boundary vectors, and transposing the auxiliary matrices $W_{s}$ and $V_{s}$. However, we have to additionally exclude all of the configurations that were captured by the tMPA for the left movers, i.e. the configurations where both the left and the right mover are emitted from the origin. Up to time $t=2$, the configurations that should be excluded 
are
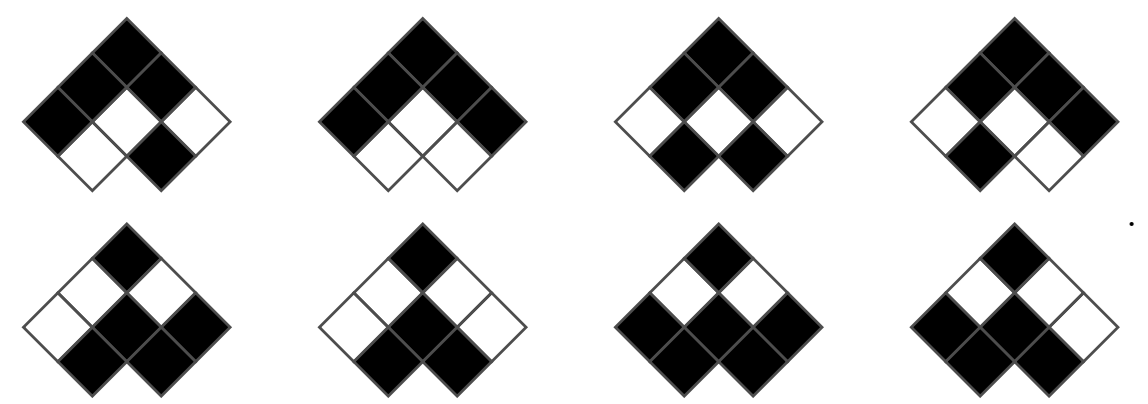

This can be achieved by considering the alternative boundary vectors

$$
\begin{aligned}
& \left|r^{\prime}(t)\right\rangle=|0, t+1,0,0\rangle, \\
& \left\langle l^{\prime}\right|=\langle 0,0,0,1|+\langle 0,0,1,1|+\langle 0,0,2,1|+\langle 0,1,0,1|+\langle 0,1,2,1|,
\end{aligned}
$$

and by changing the tMPA matrices, so that the following holds

$$
\begin{aligned}
& W_{0}^{\prime}|1,1,1,1\rangle=W_{0}^{\prime}|1,2,1,1\rangle=0, \\
& W_{1}^{\prime}|1,1,1,1\rangle=W_{1}^{\prime}|1,2,1,1\rangle=0, \\
& W_{1}^{\prime}|1,1,2,1\rangle=W_{1}^{\prime}|1,2,2,1\rangle=0, \\
& V_{0}^{\prime}|1,1,1,1\rangle=V_{0}^{\prime}|1,2,1,1\rangle=0, \\
& \left(1-P_{0}\right) V_{0}^{\prime}|1,1,1,0\rangle=0 .
\end{aligned}
$$

\section{Time-dependent density profile after inhomogeneous quench}

In this and the following section we will consider two physically relevant applications of the tMPA. The first example is an explicit calculation of the particle density profile following the inhomogeneous quench. The density profile corresponds to the probability of observing a particle at site $x$ and time $t$

$$
\hat{\rho}(x, t)=\left\langle[1]_{x}\right\rangle_{p^{t}}=\left\langle[1]_{x}^{-t}\right\rangle_{p},
$$

where the negative time propagation is given by

$$
[1]_{x}^{-t}=\left\{\begin{array}{lll}
\eta_{x}\left([1]^{t}\right) ; & x+t \equiv 1 & (\bmod 2), \\
\eta_{x}\left([1]^{t-1}\right) ; & x+t \equiv 0 & (\bmod 2) .
\end{array}\right.
$$

At time $t=0$, the system is prepared in the state 2.13), in which the probability of a site being occupied is $1 / 2$ on the left side of the chain, and 0 on the right side of the chain.

First of all note that the density profile changes only in the vicinity of the junction $-t \leq x \leq t$, and can be efficiently expressed in terms of the density profile along the 


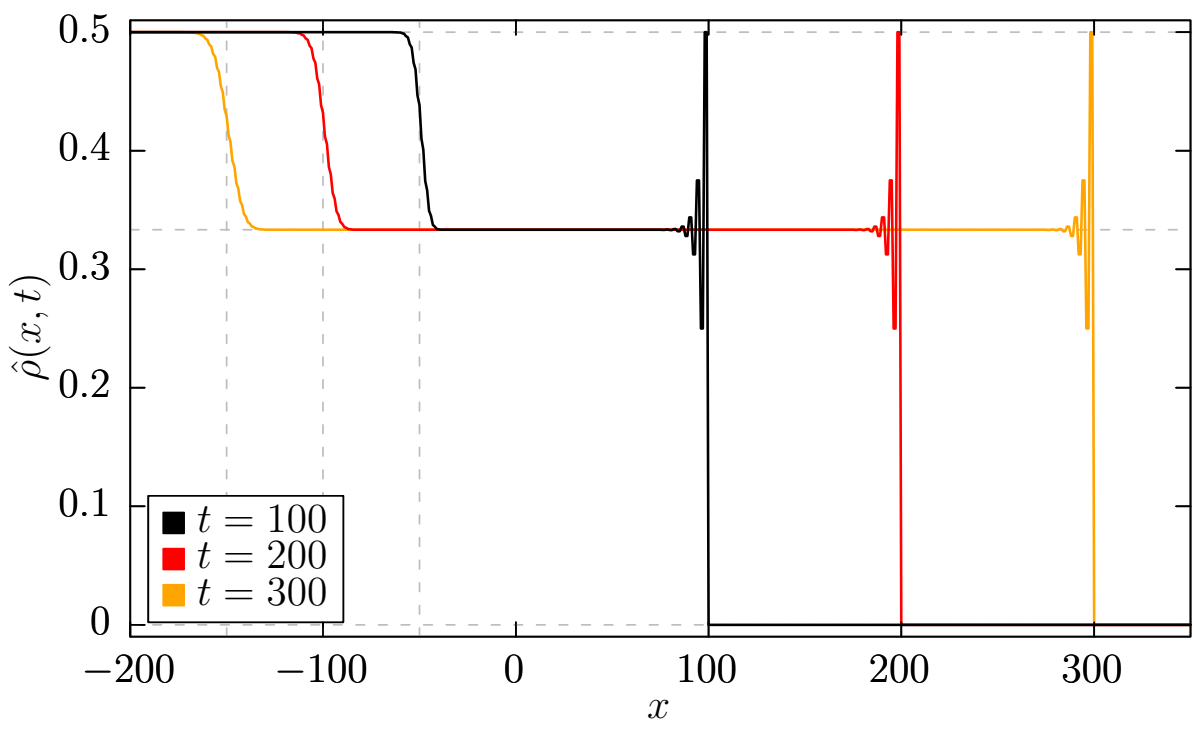

Figure 5. Density profile at different times $t$ after the quench. The ballistic front on the right moves with the velocity 1 and its shape does not change. On the left side, the profile has a shape of an erf that moves with a velocity $1 / 2$ and interpolates between $1 / 3$ towards the center and $1 / 2$ to the left. Its width scales as $\sim \sqrt{t}$.

diagonal $m, \rho(m, t)$,

$$
\hat{\rho}(x, t)= \begin{cases}0 ; & t \leq x, \\ \rho\left(\frac{t-x}{2}, t-1\right) ; & x+t \equiv 0 \quad(\bmod 2) \text { and }-t<x<t, \\ \rho\left(\frac{t-x+1}{2}, t\right) ; & x+t \equiv 1 \quad(\bmod 2) \text { and }-t<x<t, \\ \frac{1}{2} ; & x \leq-t,\end{cases}
$$

which can be calculated efficiently, using the tMPA representation

$$
\begin{aligned}
& \rho(m, t)=2^{-2 m}(L(m, t)+R(m, t)), \\
& L(m, t)=\left\langle l(t)\left|\left(\left(V_{0}+V_{1}\right)\left(W_{0}+W_{1}\right)\right)^{m} V_{0}\left(W_{0} V_{0}\right)^{t-m}\right| r\right\rangle, \\
& R(m, t)=\left\langle l^{\prime}\left|\left(\left(V_{0}^{\prime}+V_{1}^{\prime}\right)\left(W_{0}^{\prime}+W_{1}^{\prime}\right)\right)^{m} V_{0}^{\prime}\left(W_{0}^{\prime} V_{0}^{\prime}\right)^{t-m}\right| r^{\prime}(t)\right\rangle .
\end{aligned}
$$

In order to calculate the matrix elements $L(x, t)$ and $R(x, t)$, the following reduction can be employed. The action of any matrix $M_{s} \in\left\{V_{s}^{T}, V_{s}^{\prime}, W_{s}^{T}, W_{s}^{\prime}\right\}$,

(i) on a vector with the state index $a=1$ does not produce a state from the subspace corresponding to $a=0$, i.e. $P_{0} M_{s}\left(1-P_{0}\right)=0$,

(ii) on a state with $a=0$ does not increase its collision $(c)$ or its width $(w)$ index,

(iii) on the state $|0,0, n, 1\rangle$ produces a vector $|0,0, s \cdot \max \{n+1,2\}\rangle$.

Using these properties, we can calculate certain matrix elements explicitly, see Appendix 
A. In particular the density profile reads (see Appendix B),

$$
\begin{aligned}
\rho(m, t) & =\frac{3}{8} \delta_{m, t}+\frac{1}{8}\left(\delta_{m, 1} \delta_{t, 1}+\delta_{m, 2} \delta_{t, 2}\right)+\frac{1}{4} \delta_{m, 3} \delta_{t, 4}+ \\
& +\theta_{2 m-t-3} 2^{t-2 m}\left(3\left(\begin{array}{c}
2 m-t-3 \\
t-m-1
\end{array}\right)+\left(\begin{array}{c}
2 m-t-3 \\
t-m-2
\end{array}\right)\right)+ \\
& +\frac{1-\left(-\frac{1}{2}\right)^{m}}{3}-\frac{1}{2} \sum_{y=t-m}^{m-1} 2^{-(m-1-y)}\left(\begin{array}{c}
m-1-y \\
y
\end{array}\right)+ \\
& +\frac{1}{8} \sum_{y=t-m}^{2 m-t-3} 2^{-(2 m-t-3)}\left(\begin{array}{c}
2 m-t-3 \\
y
\end{array}\right)+\frac{3}{16} \sum_{y=0}^{2 m-t-4} 2^{-y}\left(\begin{array}{c}
y \\
t-m-1
\end{array}\right),
\end{aligned}
$$

where $\theta_{x}$ is a discrete Heaviside function; $\theta_{x \geq 0}=1$ and $\theta_{x<0}=0$. The profile is plotted

for three distinct times in Figure 5. From it, we can immediately identify two distinct regimes.

\subsection{Free regime}

The density profile is particularly simple in the region with the diagonal index $m \leq 2 t / 3$, where only a single term from the equation 4.5 survives,

$$
\rho\left(m \leq \frac{2 t}{3}, t\right)=\frac{1-\left(-\frac{1}{2}\right)^{m}}{3} .
$$

The density profile in this regime corresponds to the alternating exponential decay centered around $1 / 3$, traveling with a maximal velocity $v_{\max }=1$,

$$
\hat{\rho}\left(t \geq x \geq-\frac{t}{3}+1, t\right)=\frac{1}{3}\left(1-\left(-\frac{1}{2}\right)^{\left\lceil\frac{t-x}{2}\right\rceil}\right) .
$$

Note that the appearance of this regime is reminiscent of the generic situation occurring at low temperatures in any integrable model [14]. For a more intuitive understanding of this regime see Appendix C.

\subsection{Thermalizing regime}

If $m>\frac{2 t}{3}$ (and $t \geq 7$ ), the profile 4.5 can be expressed as

$$
\begin{aligned}
\rho(m, t) & =\frac{3}{8} \delta_{m, t}+2^{t-2 m-1}\left(\begin{array}{c}
2 m-t-3 \\
t-m-1
\end{array}\right)+\frac{1}{2} \sum_{y=0}^{t-m-1} 2^{-(m-1-y)}\left(\begin{array}{c}
m-1-y \\
y
\end{array}\right)+ \\
& +\frac{1}{8} \sum_{y=t-m-2}^{2 m-t-3} 2^{-(2 m-t-3)}\left(\begin{array}{c}
2 m-t-3 \\
y
\end{array}\right)+\frac{3}{16} \sum_{y=0}^{2 m-t-3} 2^{-y}\left(\begin{array}{c}
y \\
t-m-1
\end{array}\right) .
\end{aligned}
$$

Asymptotically this reduces to

$$
\lim _{t \rightarrow \infty} \rho\left(\frac{3 t}{4}+\zeta \sqrt{t}, t\right)=\frac{1}{12}(5+\operatorname{erf}(4 \zeta)),
$$




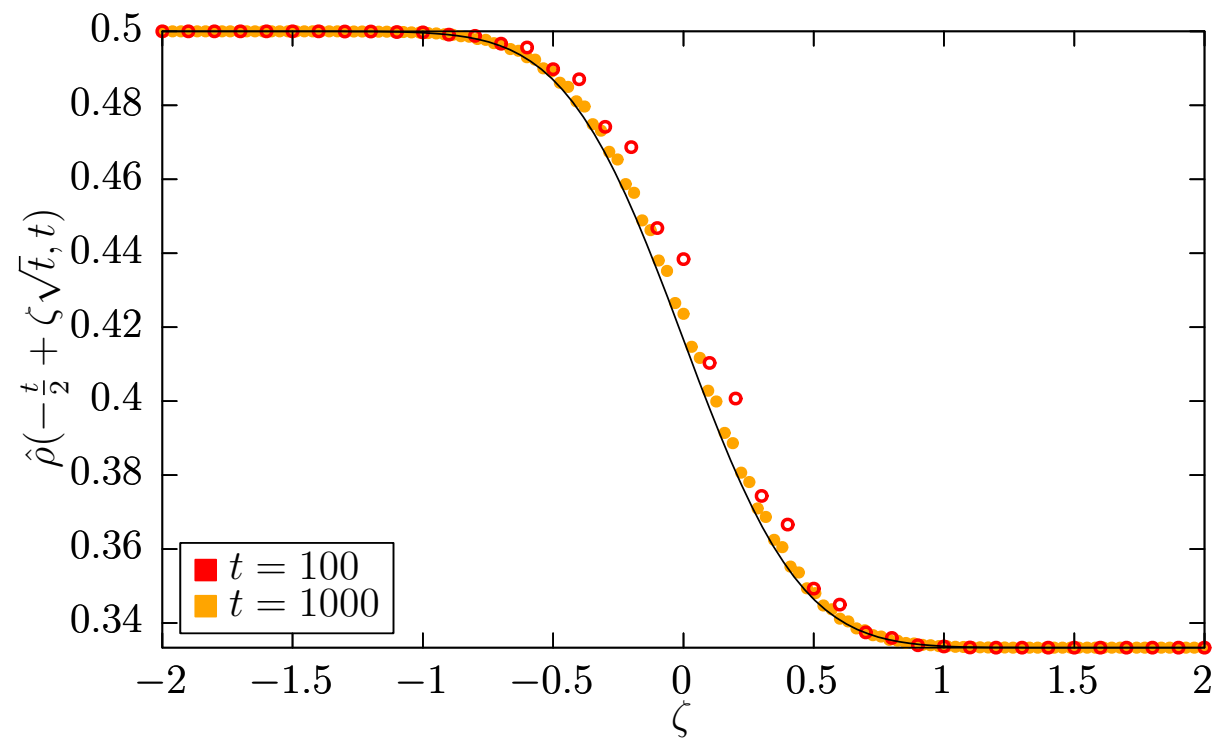

Figure 6. The density profile $\hat{\rho}(x, t)$ around $x=-\frac{t}{2}$. The solid curve denotes the asymptotic profile, $\hat{\rho}(\zeta)=\frac{1}{12}(5-\operatorname{erf}(2 \zeta))$.

implying the following shape of the density profile

$$
\hat{\rho}(\zeta) \equiv \lim _{t \rightarrow \infty} \hat{\rho}\left(-\frac{t}{2}+\zeta \sqrt{t}, t\right)=\frac{1}{12}(5-\operatorname{erf}(2 \zeta)) .
$$

The comparison of the profile at finite times and the asymptotic expression is shown in Figure 6 .

\section{Dynamic structure factor}

In this section we obtain an explicit expression of the spatio-temporal density-density correlation function, i.e. the real space-time expression for the dynamic structure factor,

$$
C(x, t)=\left\langle[1]_{x}[1]^{t}\right\rangle_{p}-\left\langle[1]_{x}\right\rangle_{p}\left\langle[1]^{t}\right\rangle_{p}=\left\langle[1]_{x}[1]^{t}\right\rangle_{p}-\frac{1}{4},
$$

where $p$ is the maximum entropy state 2.12). The dynamic structure factor corresponds to the probability that the particle, which is initially localized at the origin moves to the site $x$ in time $t$.

As a consequence of the staggered structure of the time evolution, the following holds

$$
C(x, t)=\left\{\begin{array}{lll}
C(x, t-1) ; & x+t \equiv 0 & (\bmod 2) \\
C(x, t+1) ; & x+t \equiv 1 & (\bmod 2)
\end{array}\right.
$$

implying that the generic expression for $C(x, t)$ can be obtained by considering only the cases with $x+t \equiv 0(\bmod 2)$. Under this assumption, the dynamic structure factor can be represented in terms of tMPA as

$C(x, t)=\frac{1}{2^{2 t+1}}\left(\left\langle l(t)\left|T^{\frac{x+t}{2}} V_{1} \bar{T}^{t-\frac{x+t}{2}}\right| r\right\rangle+\left\langle l^{\prime}\left|\bar{T}^{\prime \frac{x+t}{2}} V_{1}^{\prime} T^{\prime t-\frac{x+t}{2}}\right| r^{\prime}(t)\right\rangle\right)-\frac{1}{4}$, 
with

$$
\begin{array}{ll}
T=\left(V_{0}+V_{1}\right)\left(W_{0}+W_{1}\right), & \bar{T}=\left(W_{0}+W_{1}\right)\left(V_{0}+V_{1}\right), \\
T^{\prime}=\left(W_{0}^{\prime}+W_{1}^{\prime}\right)\left(V_{0}^{\prime}+V_{1}^{\prime}\right), & \bar{T}^{\prime}=\left(V_{0}^{\prime}+V_{1}^{\prime}\right)\left(W_{0}^{\prime}+W_{1}^{\prime}\right) .
\end{array}
$$

In order to simplify the derivation of the structure factor, it proves useful to consider the rescaled difference $\Delta C(x, t)$, of two next-to-nearest neighboring correlation functions,

$$
\begin{aligned}
\Delta C(x, t) & \equiv 2^{2 t+1}(C(x+2, t)-C(x, t))= \\
& =\underbrace{\left\langle l(t)\left|T^{\frac{x+t}{2}}\left(T V_{1}-V_{1} \bar{T}\right) \bar{T}^{\frac{t-x}{2}-1}\right| r\right\rangle}_{\Delta C_{l}\left(\frac{x+t}{2}, t\right)}-\underbrace{\left\langle l^{\prime}\left|\bar{T}^{\prime \frac{x+t}{2}}\left(V_{1}^{\prime} T^{\prime}-\bar{T}^{\prime} V_{1}^{\prime}\right) T^{\prime \frac{t-x}{2}-1}\right| r^{\prime}(t)\right\rangle}_{\Delta C_{r}\left(\frac{t-x}{2}-1, t\right)},
\end{aligned}
$$

where $\Delta C_{l}(m, t)$ and $\Delta C_{r}(m, t)$ correspond to the left and right movers respectively. The contributions $\Delta C_{l}(m, t)$ and $\Delta C_{r}(m, t)$ can be evaluated explicitly, see Appendix $\mathrm{D}$, yielding a following relation

$$
\Delta C(x, t)= \begin{cases}2^{2 t+x}\left(\left(\begin{array}{c}
-x-3 \\
\frac{x+t}{2}
\end{array}\right)-\left(\begin{array}{c}
-x-3 \\
\frac{x+t-2}{2}
\end{array}\right)\right), & x \leq-3, \\
0, & -2 \leq x \leq 0, \\
-2^{2 t-x-2}\left(\left(\begin{array}{c}
x-1 \\
\frac{t-x-2}{2}
\end{array}\right)-\left(\begin{array}{c}
x-1 \\
\frac{t-x-4}{2}
\end{array}\right)\right), & x \geq 1 .\end{cases}
$$

The correlation function $C(x, t)$ can be obtained recursively from $\Delta C(x, t)$, and reads

$$
\begin{aligned}
C(x, t) & =C(-t, t)+2^{-2 t-1} \sum_{m=0}^{\frac{t-|x|-2}{2}} \Delta C(-t+2 m, t)= \\
& =2^{-t-1} \sum_{m=0}^{\frac{t-|x|-2}{2}} 4^{m}\left(2\left(\begin{array}{c}
t-2 m-3 \\
m
\end{array}\right)-\left(\begin{array}{c}
t-2 m-2 \\
m
\end{array}\right)\right),
\end{aligned}
$$

where we took into account that the dynamic structure factor vanishes on the edge of the light cone, $C(-t, t)=0$. Similarly as in the case of the inhomogeneous quench problem, the structure factor can be divided into separate regimes.

\subsection{Homogeneous regime}

This regime occurs in the region $|x| \leq \frac{t}{3}+1$, where the correlation functions for $t \geq 3$ become spatially independent, save for the staggering,

$$
\begin{aligned}
& C(x, t)=2^{-t-1} c_{0}(t), \\
& c_{0}(t)=\frac{1}{4}\left(1+\frac{\mathrm{i}}{\sqrt{7}}\right)\left(\frac{-1-\mathrm{i} \sqrt{7}}{2}\right)^{t}+\frac{1}{4}\left(1-\frac{\mathrm{i}}{\sqrt{7}}\right)\left(\frac{-1+\mathrm{i} \sqrt{7}}{2}\right)^{t} .
\end{aligned}
$$

This result can be straightforwardly obtained by noting that the function $s(u)=$ $\sum_{m=0}^{\left\lfloor\frac{u}{2}\right\rfloor} 4^{m}\left(\begin{array}{c}u-2 m \\ m\end{array}\right)$ satisfies the recurrence relation $s(u)=s(u-1)-4 s(u-3)$. Solving the recurrence relation for the initial conditions $s(0)=s(1)=s(2)=1$, yields the result 5.8). Asymptotically, the correlation functions in this regime decrease as $C(x, t) \sim 1 / \sqrt{2}^{t}$. 


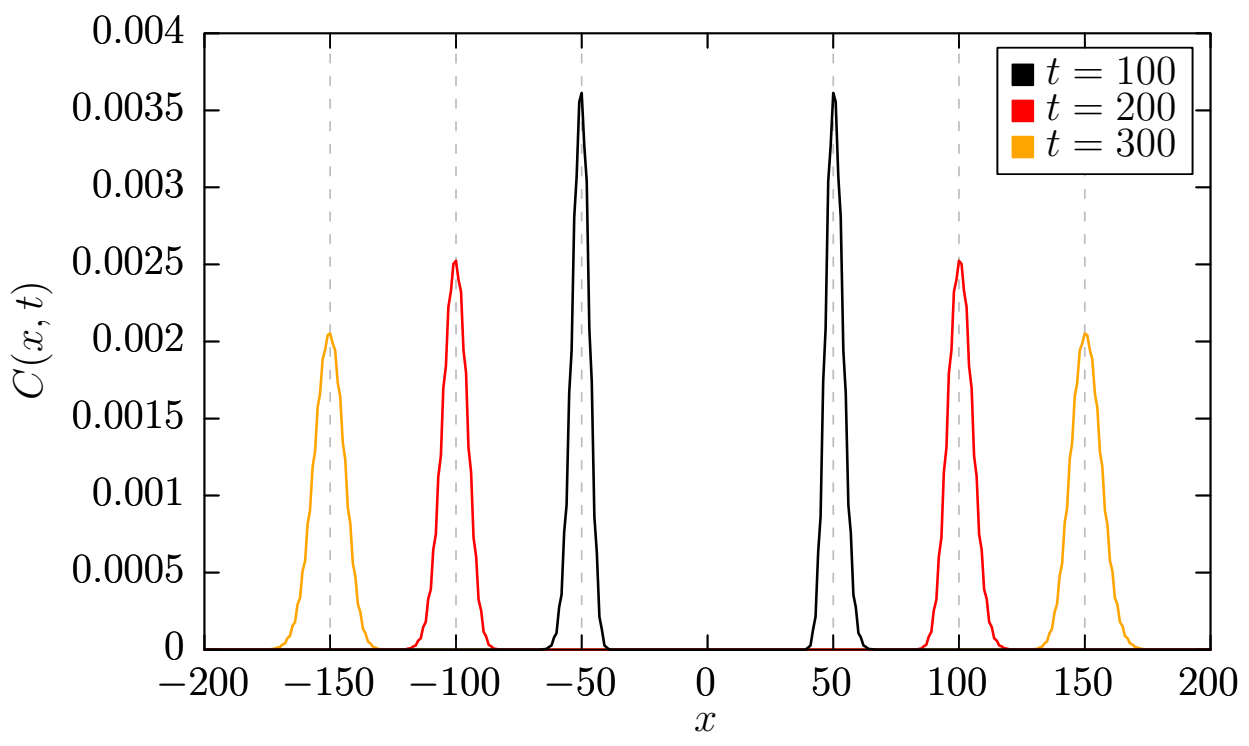

Figure 7. The dynamic structure factor $C(x, t)$ at different $t$. The two peaks move ballistically with the velocity $1 / 2$, while they spread as $\sqrt{t}$.

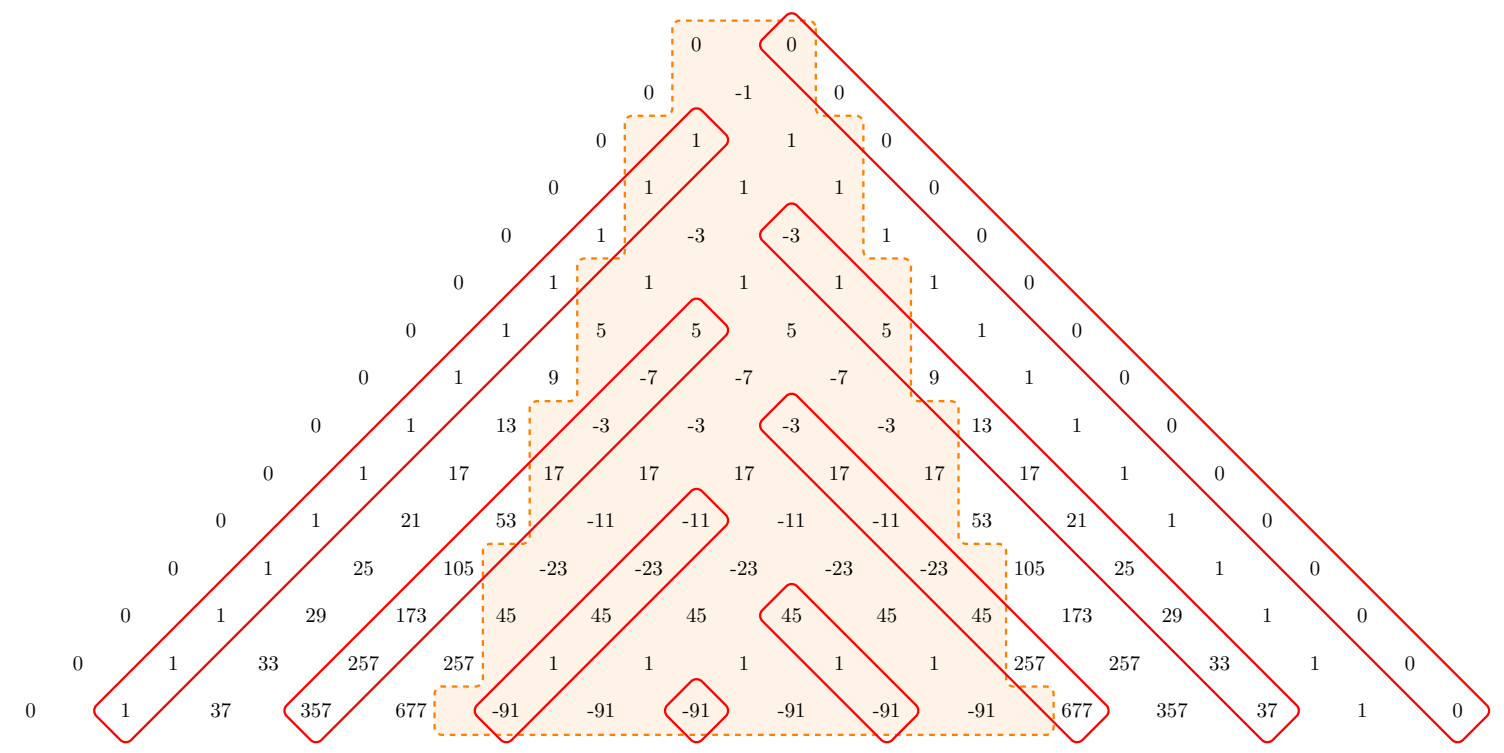

Figure 8. The explicit values of the correlations, $2^{t+1} C(x, t)$. In the shaded inner area, the correlations are homogeneous in $x$ and given by $c_{0}(t)$. Along the red bordered rays, the values are determined by polynomials of order $\frac{1}{2}(t-|x|)-1$.

\subsection{Diffusive regime}

In the diffusive regime the correlation functions $C(x, t)$ comprise of two asymptotically diffusing peaks, moving apart with a constant velocity $v= \pm \frac{1}{2}$, see Figure 7 . Let us elaborate on an explicit form of the correlation functions in this regime. Inside of the 


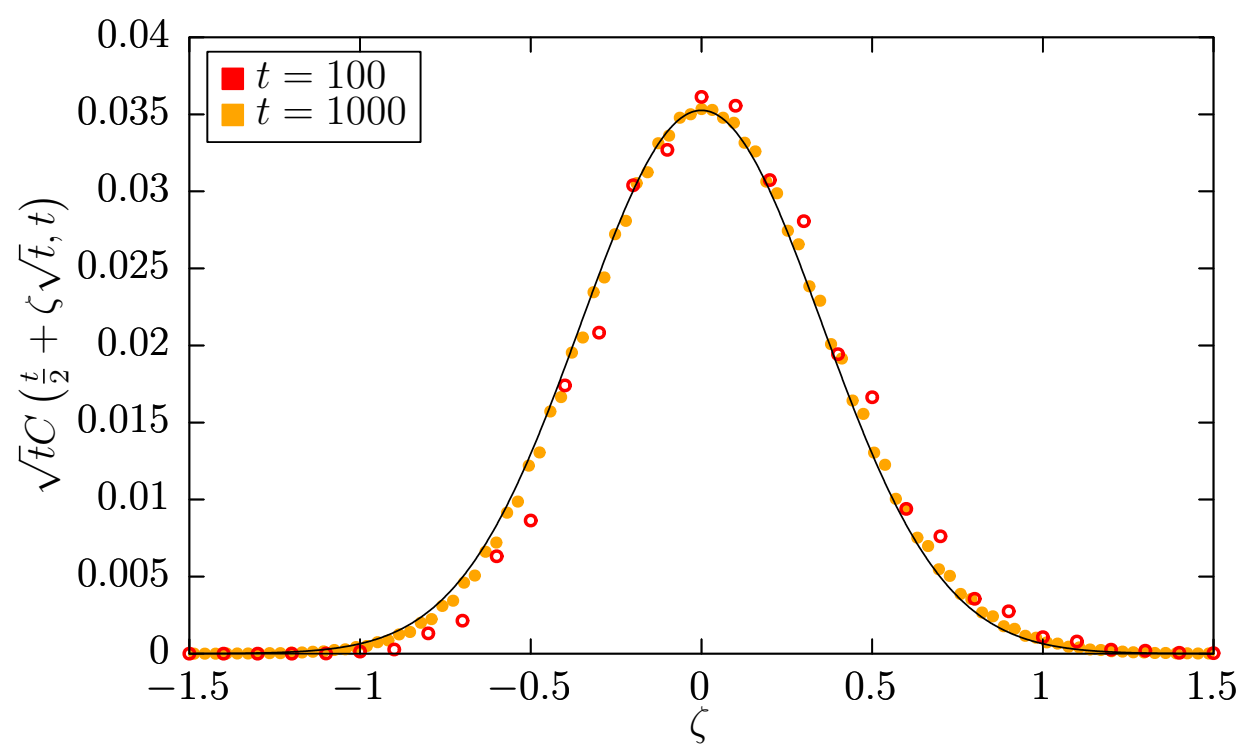

Figure 9. The correlation profile close to the peak. The solid curve denotes the asymptotic shape, $\tilde{C}(\zeta)=\frac{1}{16 \sqrt{\pi}} \mathrm{e}^{-4 \zeta^{2}}$.

region $|x| \geq \frac{t+4}{3}$, the sum (5.7) reduces to (see Appendix D.4

$$
C(x, t)=2^{-t-1} \sum_{n=t-|x|+1}^{\frac{3}{2}(t-|x|)}\left(\prod_{\substack{n=t-|x|+1 \\ n \neq j}}^{\frac{3}{2}(t-|x|)} \frac{t-j}{n-j}\right) c_{0}(n) .
$$

Along the rays with the constant distance from the edge of the light cone, the values of the reduced correlations, $2^{t+1} C(x, t)$, can be determined by the polynomial of the order $\frac{1}{2}(t-|x|-2)$ in $t$, see Figure 8. In the asymptotic regime, the peaks converge to the normal distribution (see Figure 9),

$$
C(x, t) \sim \frac{1}{16 \sqrt{t \pi}} \exp \left(-\frac{4}{t}\left(|x|-\frac{t}{2}\right)^{2}\right) .
$$

With this we close the discussion of the tMPA construction and its applications.

\section{Conclusions and discussion}

In this article we constructed explicit time evolution of local observables in terms of the tMPA for a deterministic interacting lattice gas, specifically RCA 54. The auxiliary matrices encode the backward propagation of solitons, where the auxiliary boundary vectors select the states with a soliton originating from the center of the chain.

In the second part of the article the tMPA was applied in order to provide analytical time-dependent solutions of two out-of-equilibrium setups. In the first one we considered the time-dependent density profile arising from a piecewise homogeneous initial state, with the maximum entropy (half-filled) state on the left side of the chain and the empty lattice on the right side of the chain. In the light-cone around the origin two distinct 
regions emerge. On the right side the dynamics reduces to the sea of non-interacting right movers, implying regime with exponential decay of the density profile with respect to the spatial coordinate. To the left of the non-interacting sea a thermalizing region occurs as a consequence of the interactions of solitons, causing an emergence of the diffusive error function shaped density profile with the center moving at a constant velocity.

In the second setup we calculated the dynamical structure factor, which is a hallmark of the transport phenomena. The structure factor supports two regimes. In the central region a spatially homogeneous state is formed, while the values outside of the central regime are determined by polynomials along the diagonals. Asymptotically, the correlations behave as two diffusively spreading Gaussian peaks moving with a constant velocity. Both of the results analytically demonstrate the coexistence of the ballistic and diffusive transport.

We used the maximum entropy state for our calculations, which is invariant under time translations, and represents a caricature of a high-temperature state in physics. We believe that our explicit computations should be generalizable to a larger family of invariant clustering states which are described in terms of $4 \times 4$ transfer matrices with two free spectral parameters $\xi, \omega[9,11$, specifically $p_{\cdots s_{-2}, s_{-1}, s_{0}, s_{1}, s_{2}, s_{3} \cdots}=\cdots T_{s_{-2} s_{-1}, s_{0} s_{1}}(\xi, \omega) T_{s_{0} s_{1}, s_{2} s_{3}}(\omega, \xi) T_{s_{2} s_{3}, s_{4} s_{5}}(\xi, \omega) \cdots$, where $\xi, \omega$ should be real and positive and

$$
T(\xi, \omega)=\left[\begin{array}{cccc}
1 & 1 & \xi & 1 \\
\xi \omega & \xi \omega & 1 & \omega \\
\omega & \omega & \xi \omega & \omega \\
\xi & \xi & \xi & \xi \omega
\end{array}\right] .
$$

Note that the maximum entropy (separable) state corresponds to $\xi=\omega=1$.

The research presented in this paper opens several interesting questions, both from the mathematical as well as physical perspective.

(i) The first question is what is the type of models for which we can obtain explicit time dependent representation of local observables in terms of the tMPA. Namely, the results presented in this paper go beyond what is currently possible in generic integrable models. At this point we conjecture that such a solution should be attainable for any purely solitonic, deterministic model with discrete space-time dynamics. This line of thought is supported by the tMPA solution of the somewhat simpler model of hard-core interacting charged particles [15] (see [16] for an explicit construction of the tMPA). We hope that our approach might offer some insight into more complicated systems, for example the discrete space-time versions of the exclusion processes [17, 18, or even space-time discrete quantum integrable models $[19$.

(ii) The second question is how far the explicit tMPA parametrization can push our knowledge regarding the fluctuations in solitonic models. Ideally, one could derive a complete large deviation functional for the types of the models considered in the 
present paper, and explicitly monitor the validity of the macroscopic fluctuation theory [20].

(iii) Furthermore, the origins of, and explicit algebraic structure behind integrability of the model presented in this paper remain largely unexplored. This question is naturally linked to the first one.

(iv) Finally, it would be worthwhile studying the tMPA solvable models exhibiting different types of transport behavior [21, 22], since they could provide the insight into the microscopic roots of different transport universality classes.

\section{Acknowledgements}

The work has been supported by Advanced Grant 694544 - OMNES of European Research Council (ERC), and by Research program P1-0044 of Slovenian Research Agency (ARRS).

\section{References}

[1] Spohn H 2012 Large scale dynamics of interacting particles (Springer Science \& Business Media)

[2] Golse F 2013 Scholarpedia 89141 revision \#136985 http://www.scholarpedia.org/article/ Boltzmann-Grad_limit

[3] Castro-Alvaredo O A, Doyon B and Yoshimura T 2016 Phys. Rev. X 6(4) 041065 https: //link.aps.org/doi/10.1103/PhysRevX.6.041065

[4] Bertini B, Collura M, De Nardis J and Fagotti M 2016 Phys. Rev. Lett. 117(20) 207201 https://link.aps.org/doi/10.1103/PhysRevLett.117.207201

[5] Doyon B, Spohn H and Yoshimura T 2018 Nucl. Phys. B 926 570-583 https://doi.org/10. 1016/j.nuclphysb.2017.12.002

[6] De Nardis J, Bernard D and Doyon B 2018 arXiv preprint arXiv:1807.02414 https://arxiv. org/abs/1807.02414

[7] Bobenko A, Bordemann M, Gunn C and Pinkall U 1993 Commun. Math. Phys. 158 127-134 https://doi.org/10.1007/BF02097234

[8] Takesue S 1987 Phys. Rev. Lett. 59(22) 2499-2502 https://link.aps.org/doi/10.1103/ PhysRevLett.59.2499

[9] Prosen T and Mejía-Monasterio C 2016 J. Phys. A: Math. Theor. 49185003 http://stacks. iop.org/1751-8121/49/i=18/a=185003

[10] Inoue A and Takesue S 2018 arXiv preprint arXiv:1806.07099 https://arxiv.org/abs/1806. 07099

[11] Prosen T and Buča B 2017 J. Phys. A: Math. Theor. 50395002 http://stacks.iop.org/ $1751-8121 / 50 / i=39 / a=395002$

[12] Gopalakrishnan S 2018 arXiv preprint arXiv:1806.04156 https://arxiv.org/abs/1806.04156

[13] Bratteli O and Robinson D W 1997 Operator Algebras and Quantum Statistical Mechanics: Volume 18 Volume 2, Second Edition (Springer Science \& Business Media)

[14] Bertini B and Piroli L 2018 J. Stat. Mech. 2018033104 http://stacks.iop.org/1742-5468/ $2018 / i=3 / a=033104$

[15] Medenjak M, Klobas K and Prosen T 2017 Phys. Rev. Lett. 119(11) 110603 https://link.aps. org/doi/10.1103/PhysRevLett.119.110603

[16] Medenjak M 2018 Quasilocality and equilibration in quantum systems Ph.D. thesis University of Ljubljana 
[17] Rajewsky N, Santen L, Schadschneider A and Schreckenberg M 1998 J. Stat. Phys. 92 151-194 https://doi.org/10.1023/A:1023047703307

[18] Vanicat M 2018 Nucl. Phys. B 929 298-329 http://www.sciencedirect.com/science/ article/pii/S0550321318300464

[19] Vanicat M, Zadnik L and Prosen T 2017 arXiv preprint arXiv:1712.00431 https://arxiv.org/ abs/1712.00431

[20] Bertini L, De Sole A, Gabrielli D, Jona-Lasinio G and Landim C 2015 Rev. Mod. Phys. 87(2) 593-636 https://link.aps.org/doi/10.1103/RevModPhys.87.593

[21] Popkov V, Schadschneider A, Schmidt J and Schütz G M 2015 Proc. Natl. Acad. Sci. USA 112 12645-12650 http://www.pnas.org/content/112/41/12645

[22] Ilievski E, De Nardis J, Medenjak M and Prosen T 2018 arXiv preprint arXiv:1806.03288 https://arxiv.org/abs/1806.03288

\section{Appendix A. The action of the matrices $T, T^{\prime}, \bar{T}$ and $\bar{T}^{\prime}$}

In this appendix we explicitly compute the powers of the matrices $T$ and $\bar{T}\left(T^{\prime}\right.$ and $\left.\bar{T}^{\prime}\right)$ acting onto the left (right), with the matrices defined as

$$
\begin{array}{ll}
T=\left(V_{0}+V_{1}\right)\left(W_{0}+W_{1}\right), & \bar{T}=\left(W_{0}+W_{1}\right)\left(V_{0}+V_{1}\right), \\
T^{\prime}=\left(W_{0}^{\prime}+W_{1}^{\prime}\right)\left(V_{0}^{\prime}+V_{1}^{\prime}\right), & \bar{T}^{\prime}=\left(V_{0}^{\prime}+V_{1}^{\prime}\right)\left(W_{0}^{\prime}+W_{1}^{\prime}\right),
\end{array}
$$

but first let us discuss the general structure of the matrices $M \in\left\{\alpha V_{0}+\beta V_{1}, \alpha W_{0}+\right.$ $\left.\beta W_{1} ; \alpha, \beta \in \mathbb{R}\right\}$ and $M^{\prime} \in\left\{\alpha V_{0}^{\prime}+\beta V_{1}^{\prime}, \alpha W_{0}^{\prime}+\beta W_{1}^{\prime} ; \alpha, \beta \in \mathbb{R}\right\}$. We start by defining the projectors to the subspace of "unactivated" vectors, i.e. the subspace defined by $a=0$, the subspace of "activated" vectors with width $0(a=1$ and $w=0)$ and to the subspace of vectors with $a=1$ and $w \leq 1$,

$$
\begin{aligned}
P_{0}|c, w, n, a\rangle & =\delta_{a, 0}|c, w, n, 0\rangle \\
Q|c, w, n, a\rangle & =\delta_{a, 1} \delta_{w, 0}|c, 0, n, 1\rangle \\
Q^{\prime}|c, w, n, a\rangle & =\delta_{a, 1} \delta_{w, 0}|c, 0, n, 1\rangle+\delta_{a, 1} \delta_{w, 1}|c, 1, n, 1\rangle .
\end{aligned}
$$

The subspace with $a=1$ is invariant to multiplication by matrices $M^{T}$ and $M^{\prime}$,

$$
P_{0} M^{T} P_{0}=P_{0} M^{T}, \quad P_{0} M^{\prime} P_{0}=P_{0} M^{\prime},
$$

and the value of $w$ inside the $a=1$ subspace cannot increase, which implies the following,

$$
\begin{array}{ll}
Q M^{T} Q=M^{T} Q, & Q M^{\prime} Q=M^{\prime} Q, \\
Q^{\prime} M^{T} Q^{\prime}=M^{T} Q^{\prime}, & Q^{\prime} M^{\prime} Q^{\prime}=M^{\prime} Q^{\prime} .
\end{array}
$$

Additionally, the matrices $M\left(M^{\prime}\right)$ commute with the raising/lowering operators defined in the main text equation (3.6) as long as $w \geq 1(w \geq 2)$. Explicitly,

$$
\begin{aligned}
& (1-Q)\left[\mathbf{c}^{ \pm}, M^{T}\right]=0, \quad(1-Q)\left[\mathbf{w}^{ \pm}, M^{T}\right]=0, \\
& \left(1-Q^{\prime}\right)\left[\mathbf{c}^{ \pm}, M^{\prime}\right]=0, \quad\left(1-Q^{\prime}\right)\left[\mathbf{w}^{ \pm}, M^{\prime}\right]=0 .
\end{aligned}
$$

We wish to obtain $\langle v| T^{x},\langle v| \bar{T}^{x}$ (or $\left.T^{\prime x}|v\rangle, \bar{T}^{x}|v\rangle\right)$ for an arbitrary vector $|v\rangle \in \mathcal{V}$. Due to the mentioned properties, it is convenient to first express the $w \geq 1$ (or $w \geq 2$ ) projections,

$$
\begin{array}{ll}
\langle v| T^{x}(1-Q), & \langle v| \bar{T}^{x}(1-Q), \\
\left(1-Q^{\prime}\right) T^{\prime x}|v\rangle, & \left(1-Q^{\prime}\right) \bar{T}^{\prime x}|v\rangle,
\end{array}
$$


and compute the relevant overlaps using these vectors (for specific examples see equations (B.4), (B.17), (D.1) and (D.2)).

Furthermore, the matrices $V_{s}^{T}, W_{s}^{T}$ and $V_{s}^{\prime}, W_{s}^{\prime}$ differ only in the boundary terms; explicitly,

$$
\left(1-Q^{\prime}\right)\left(V_{s}^{\prime}-V_{s}^{T}\right)=0, \quad\left(1-Q^{\prime}\right)\left(W_{s}^{\prime}-W_{s}^{T}\right)=0,
$$

therefore we can express the products of right-soliton matrices by projecting the corresponding left-soliton products to the subspace with $w \geq 2$ and transpose them,

$$
\begin{aligned}
& \left(1-Q^{\prime}\right) T^{\prime x}|v\rangle=\left(\langle v| T^{x}(1-Q)\left(1-Q^{\prime}\right)\right)^{T}, \\
& \left(1-Q^{\prime}\right) \bar{T}^{\prime x}|v\rangle=\left(\langle v| \bar{T}^{x}(1-Q)\left(1-Q^{\prime}\right)\right)^{T} .
\end{aligned}
$$

Thus, it suffices to express $\langle v| T^{x}(1-Q)$ and $\langle v| \bar{T}^{x}(1-Q)$.

Appendix A.1. The powers $T^{m}$

The matrices $W_{s}, V_{s}$ restricted to the subspace with $a=0$ are simple, as are $T$ and $\bar{T}$,

$$
\begin{aligned}
\langle c, w, n, 0| T P_{0} & =\langle c, w, n, 0| \bar{T} P_{0}= \\
& =2\langle c+1, w-1,0,0|+\langle c+1, w-1,1,0|+\langle c+1, w-1,2,0| .
\end{aligned}
$$

Since the subspace with $a=1$ is an invariant subspace of the left action of matrices $V_{s}$, $W_{s}$, the following holds

$$
\begin{aligned}
\langle c, w, n, 0| T^{x} P_{0} & =\langle c, w, n, 0| \bar{T}^{x} P_{0}= \\
& =4^{x-1}(2\langle c+x, w-x, 0,0|+\langle c+x, w-x, 1,0|+\langle c+x, w-x, 2,0|),
\end{aligned}
$$

as long as $x \leq w$, otherwise the r.h.s. is 0 .

Now let us focus on the subspace spanned by $\{|c, w, n, 1\rangle ; c \geq 0, w>0, n \in$ $\{0,1,2\}\}$. Due to the value of $w$ and $c$ decreasing, it is convenient to express the left action of $T^{x}$ to the basis vectors $\langle c, w, n, 1|$ in the following form

$$
\begin{aligned}
\langle c, w, n, 1| T^{x}(1-Q) & =\sum_{m, p} f_{x}^{n}(m, p)\langle c-m, w-x-p, 0,1|+ \\
& +\sum_{m, p} g_{x}^{n}(m, p)\langle c-m, w-x-p, 1,1|+ \\
& +\sum_{m, p} h_{x}^{n}(m, p)\langle c-m, w-x-p, 2,1|,
\end{aligned}
$$

where $f_{x}^{n}, g_{x}^{n}, h_{x}^{n}$ are some unknown coefficients that have to satisfy the following recurrence relation

$$
\begin{aligned}
f_{x+1}^{n}(m, p) & =f_{x}^{n}(m, p)+f_{x}^{n}(m-1, p-1)+g_{x}^{n}(m, p-1)+ \\
& +g_{x}^{n}(m-1, p-1)+h_{x}^{n}(m, p)+h_{x}^{n}(m, p-1), \\
g_{x+1}^{n}(m, p) & =f_{x}^{n}(m, p)+g_{x}^{n}(m-1, p-1)+h_{x}^{n}(m, p), \\
h_{x+1}^{n}(m, p) & =f_{x}^{n}(m-1, p)+g_{x}^{n}(m-1, p-1)+h_{x}^{n}(m-1, p-1) .
\end{aligned}
$$


A family of solutions is parametrized by 4 parameters, $\alpha, \beta, \gamma$ and $\delta$,

$$
\begin{aligned}
& f_{x}(m, p)=\left(\begin{array}{c}
x-m+p+\alpha \\
m+\beta
\end{array}\right)\left(\begin{array}{c}
x+m-p+\gamma \\
p+\delta
\end{array}\right), \\
& g_{x}(m, p)=\left(\begin{array}{c}
x-m+p+\alpha \\
m+\beta
\end{array}\right)\left(\begin{array}{c}
x+m-p-1+\gamma \\
p+\delta
\end{array}\right), \\
& h_{x}(m, p)=\left(\begin{array}{c}
x-m+p+\alpha \\
m-1+\beta
\end{array}\right)\left(\begin{array}{c}
x+m-p-1+\gamma \\
p+\delta
\end{array}\right) .
\end{aligned}
$$

Taking into account the appropriate initial conditions, it is possible to express the coefficients $f_{x}^{n}, g_{x}^{n}, h_{x}^{n}$ in terms of this solution with the following parametrization,

$$
\begin{array}{ll}
n=0: & (\alpha, \beta, \gamma, \delta)=(0,0,0,0), \\
n=1: & (\alpha, \beta, \gamma, \delta)=(0,0,0,-1), \\
n=2: & (\alpha, \beta, \gamma, \delta)=(-1,0,1,0) .
\end{array}
$$

Now we are almost able to express the whole $\langle v| T^{x}(1-Q)$ for any vector $\langle v|$. The last remaining property is

$$
\begin{aligned}
\langle c, w, 0,0| T\left(1-P_{0}\right) & =\langle c, w-1,0,1|, \\
\langle c, w, 1,0| T\left(1-P_{0}\right) & =\langle c, w-1,0,1|+\langle c-1, w-1,0,1|+ \\
& +\langle c-1, w-1,1,1|+\langle c-1, w-1,2,1|, \\
\langle c, w, 2,0| T\left(1-P_{0}\right) & =\langle c, w-1,0,1|+\langle c-1, w-1,2,1| .
\end{aligned}
$$

Combining the equations A.10 and A.15 with the expressions A.13), and A.14, we can explicitly obtain the coefficients in the basis expansion of $\langle v| T^{x}(1-Q)$ in terms of sums of coefficients A.13. For sufficiently simple $\langle v|$ they simplify, as for example in the case $\langle v|=\langle 0, t, 0,0|=\langle l(t)|$,

$$
\begin{aligned}
&\langle l(t)| T^{x}(1-Q)=4^{x-1}(2\langle x, t-x, 0,0|+\langle x, t-x, 1,0|+\langle x, t-x, 2,0|)+ \\
&+\sum_{m=0}^{x} \sum_{p=0}^{\min \{m-1, t-x-1\}} \mathcal{A}_{x}^{0}(m, p)\langle x-m, t-x-p, 0,1|+ \\
&+\sum_{m=0}^{x} \sum_{p=0}^{\min \{m-2, t-x-1\}} \mathcal{A}_{x}^{1}(m, p)\langle x-m, t-x-p, 1,1|+ \\
&+\sum_{m=0}^{x} \sum_{p=0}^{\min \{m-2, t-x-1\}} \mathcal{A}_{x}^{2}(m, p)\langle x-m, t-x-p, 2,1| ; \\
& \mathcal{A}_{x}^{0}(m, p)=2^{2 x+p-m-1}\left(\begin{array}{c}
m-p-1 \\
p
\end{array}\right), \quad \mathcal{A}_{x}^{1,2}(m, p)=2^{2 x+p-m-1}\left(\begin{array}{c}
m-p-2 \\
p
\end{array}\right) .
\end{aligned}
$$


Appendix A.2. The powers $\bar{T}^{m}$

Similarly, the left action of $\bar{T}^{x}$ on $\langle c, w, n, 1|$ can be expressed in terms of basis vectors via coefficients $\bar{f}_{x}^{n}, \bar{g}_{x}^{n}, \bar{h}_{x}^{n}$ as

$$
\begin{aligned}
\langle c, w, n, 1| T^{x}(1-Q) & =\sum_{m, p} \bar{f}_{x}^{n}(m, p)\langle c-m, w-x-p, 0,1|+ \\
& =\sum_{m, p} \bar{g}_{x}^{n}(m, p)\langle c-m, w-x-p, 1,1|+ \\
& =\sum_{m, p} \bar{h}_{x}^{n}(m, p)\langle c-m, w-x-p, 2,1|,
\end{aligned}
$$

with the coefficients satisfying a recurrence relation similar to A.12,

$$
\begin{aligned}
\bar{f}_{x+1}^{n}(m, p) & =\bar{f}_{x}^{n}(m, p)+\bar{f}_{x}^{n}(m-1, p-1)+\bar{g}_{x}^{n}(m-1, p)+ \\
& +\bar{g}_{x}^{n}(m-1, p-1)+\bar{h}_{x}^{n}(m, p)+\bar{h}_{x}^{n}(m-1, p), \\
\bar{g}_{x+1}^{n}(m, p) & =\bar{f}_{x}^{n}(m, p)+\bar{g}_{x}^{n}(m-1, p-1)+\bar{h}_{x}^{n}(m, p), \\
\bar{h}_{x+1}^{n}(m, p) & =\bar{f}_{x}^{n}(m, p-1)+\bar{g}_{x}^{n}(m-1, p-1)+\bar{h}_{x}^{n}(m-1, p-1) .
\end{aligned}
$$

Again, a family of solutions is parametrized by 4 parameters,

$$
\begin{aligned}
& \bar{f}_{x}(m, p)=\left(\begin{array}{c}
x-m+p+\alpha \\
m+\beta
\end{array}\right)\left(\begin{array}{c}
x+m-p+\gamma \\
p+\delta
\end{array}\right), \\
& \bar{g}_{x}(m, p)=\left(\begin{array}{c}
x-m+p-1+\alpha \\
m+\beta
\end{array}\right)\left(\begin{array}{c}
x+m-p+\gamma \\
p+\delta
\end{array}\right), \\
& \bar{h}_{x}(m, p)=\left(\begin{array}{c}
x-m+p-1+\alpha \\
m+\beta
\end{array}\right)\left(\begin{array}{c}
x+m-p+\gamma \\
p-1+\delta
\end{array}\right),
\end{aligned}
$$

and the values of parameters corresponding to particular solutions $\bar{f}_{x}^{n}, \bar{g}_{x}^{n}, \bar{h}_{x}^{n}$ are

$$
\begin{array}{ll}
n=0: & (\alpha, \beta, \gamma, \delta)=(0,0,0,0), \\
n=1: & (\alpha, \beta, \gamma, \delta)=(0,-1,0,0), \\
n=2: & (\alpha, \beta, \gamma, \delta)=(1,0,-1,0) .
\end{array}
$$

The relation equivalent to A.15 is

$\langle c, w, 0,0| \bar{T}\left(1-P_{0}\right)=\langle c, w-1,0,1|+\langle c+1, w-1,2,1|$,

$\langle c, w, 1,0| \bar{T}\left(1-P_{0}\right)=\langle c, w-1,0,1|+\langle c, w-1,1,1|+\langle c+1, w-1,2,1|$,

$\langle c, w, 2,0| \bar{T}\left(1-P_{0}\right)=\langle c+1, w-1,2,1|$.

As before, it is possible to explicitly express $\langle v| \bar{T}^{x}$ in terms of sums of coefficients $f_{x}^{n}$, $g_{x}^{n}, h_{x}^{n}$ for any vector $\langle v|$. For some special vectors, the expressions are simple. For 
example,

$$
\begin{aligned}
& \langle c, w, 0,0| \bar{T}^{x}(1-Q)=4^{x-1}(2\langle c+x, w-x, 0,0|+\langle c+x, w-x, 1,0|+ \\
& +\langle c+x, w-x, 2,0|+\langle c+x, w-x, 2,1|)+ \\
& \min \{c+x-1,2 x-1\} \min \{w-x-1, m-1\} \\
& +\sum_{m=1} \quad \sum_{p=0} \overline{\mathcal{A}}_{x}^{0}(m, p)\langle c+x-m, w-x-p, 0,1|+ \\
& \min \{c+x-1,2 x-1\} \min \{w-x-1, m-1\} \\
& +\sum_{m=1} \quad \sum_{p=0} \overline{\mathcal{A}}_{x}^{1}(m, p)\langle c+x-m, w-x-p, 1,1|+ \\
& \min \{c+x-1,2 x-1\} \min \{w-x-1, m-1\} \\
& +\sum_{m=1} \quad \sum_{p=1} \overline{\mathcal{A}}_{x}^{2}(m, p)\langle c+x-m, w-x-p, 2,1|
\end{aligned}
$$

with the coefficients $\overline{\mathcal{A}}_{x}^{n}$ defined as

$$
\begin{aligned}
& \overline{\mathcal{A}}_{x}^{0}(m, p)=\left(\begin{array}{c}
m-p-1 \\
p
\end{array}\right) \sum_{y=m-x}^{2 x+p-m-1}\left(\begin{array}{c}
2 x+p-m-1 \\
y
\end{array}\right), \\
& \overline{\mathcal{A}}_{x}^{1}(m, p)=\left(\begin{array}{c}
m-p-1 \\
p
\end{array}\right) \sum_{y=m-x}^{2 x+p-m-2}\left(\begin{array}{c}
2 x+p-m-2 \\
y
\end{array}\right), \\
& \overline{\mathcal{A}}_{x}^{2}(m, p)=\left(\begin{array}{c}
m-p-1 \\
p-1
\end{array}\right) \sum_{y=m-x}^{2 x+p-m-2}\left(\begin{array}{c}
2 x+p-m-2 \\
y
\end{array}\right) .
\end{aligned}
$$

Note that we assumed $w \geq x$.

\section{Appendix B. The inhomogeneous quench}

We wish to explicitly obtain the overlaps

$$
\begin{aligned}
& L(x, t)=\left\langle l(t)\left|T^{x} V_{0} U^{t-x}\right| r\right\rangle, \\
& R(x, t)=\left\langle l^{\prime}\left|\bar{T}^{\prime x} V_{0}^{\prime} U^{\prime-x}\right| r^{\prime}(t)\right\rangle,
\end{aligned}
$$

with $0 \leq x \leq t$ and

$$
\begin{array}{ll}
T=\left(V_{0}+V_{1}\right)\left(W_{0}+W_{1}\right), & U=W_{0} V_{0}, \\
\bar{T}^{\prime}=\left(V_{0}^{\prime}+V_{1}^{\prime}\right)\left(W_{0}^{\prime}+W_{1}^{\prime}\right), & U^{\prime}=W_{0}^{\prime} V_{0}^{\prime} .
\end{array}
$$

Let us start with the overlap that corresponds to the left moving solitons.

Appendix B.1. Expressing the overlap $L(x, t)$

The matrices $V_{s}, W_{s}$ act trivially on the vectors $\langle 0,0, n, 1|$,

$$
\begin{aligned}
& \langle 0,0, n, 1| V_{s}=\langle 0,0, n, 1| W_{s}=\langle 0,0, s \cdot \max \{n+1,2\}, 1|, \\
& \langle 0,0, n, 1| T=2\langle 0,0,0,1|+\langle 0,0,1,1|+\langle 0,0,2,1|,
\end{aligned}
$$

therefore we can treat these vectors separately. Since they are the only vectors with the nonzero overlap with $|r\rangle$, computing $L(x, t)$ is equivalent to summing up the 
contributions of $\langle 0,0, n, 1|$ vectors that are created at different steps. Explicitly,

$$
\begin{aligned}
L(x, t) & =\underbrace{\left(1-\delta_{x, 0}\right)\langle l(t)|T| r\rangle}_{\equiv L_{1}(x, t)}+\underbrace{\sum_{y=1}^{x} 4^{x-y}\left\langle l(t)\left|T^{y-1}(1-Q) T\right| r\right\rangle}_{\equiv L_{2}(x, t)}+ \\
& +\underbrace{\left\langle l(t)\left|T^{x}(1-Q) V_{0}\right| r\right\rangle+\sum_{z=1}^{t-x-1}\left\langle l(t)\left|T^{x} V_{0} U^{z-1}(1-Q) U\right| r\right\rangle}_{\equiv L_{3}(x, t)} .
\end{aligned}
$$

Since $L(0, t)=0$, let us from now on assume $x>0$ to simplify the notation. The first contribution is easy; if $t \neq 0$, the only nonzero overlap occurs for $x=t=1$,

$$
L_{1}(x, t)=\delta_{x, 1} \delta_{t, 1} .
$$

The second contribution $L_{2}(x, t)$ is obtained from A.16 as

$$
\begin{aligned}
& L_{2}(x, t)=4 \delta_{x, 2} \delta_{t, 2}+\sum_{y=\left\lceil\frac{t+2}{2}\right\rceil}^{x-1} 2 \cdot 4^{x-y-1} \mathcal{A}_{y}^{1}(y-1, t-y-1)+ \\
& +\sum_{y=\left\lceil\frac{t+1}{2}\right\rceil}^{x-1} 4^{x-y-1}\left(2 \mathcal{A}_{y}^{0}(y-1, t-y-1)+6 \mathcal{A}_{y}^{1}(y, t-y-1)+4 \mathcal{A}_{y}^{1}(y-1, t-y-2)\right)+( \\
& +\sum_{y=\left\lceil\frac{t}{2}\right\rceil}^{x-1} 4^{x-y-1}\left(2 \mathcal{A}_{y}^{0}(y, t-y-1)+\mathcal{A}_{y}^{0}(y-1, t-y-2)+2 \mathcal{A}_{y}^{1}(y, t-y-2)\right) .
\end{aligned}
$$

Taking into account the form of the coefficients from A.16 the first two contributions combine into

$$
\begin{aligned}
& \begin{aligned}
L_{1}(x, t)+L_{2}(x, t) & =2^{2 x-t-1}\left(\sum_{z=t-x}^{\left\lfloor\frac{t-1}{2}\right\rfloor} 4^{z}\left(\begin{array}{c}
t-1-2 z \\
z
\end{array}\right)+2 \sum_{z=t-x}^{\left\lfloor\frac{t-2}{2}\right\rfloor} 4^{z}\left(\begin{array}{c}
t-2-2 z \\
z
\end{array}\right)+\right. \\
& \left.+3 \sum_{z=t-x}^{\left\lfloor\frac{t-3}{2}\right\rfloor} 4^{z}\left(\begin{array}{c}
t-3-2 z \\
z
\end{array}\right)+2 \sum_{z=t-x}^{\left\lfloor\frac{t-4}{2}\right\rfloor} 4^{z}\left(\begin{array}{c}
t-4-2 z \\
z
\end{array}\right)\right)=
\end{aligned} \\
& =2^{2 x}\left(\frac{1}{4} u(t-1, t-x)+\frac{1}{4} u(t-2, t-x)+\frac{3}{16} u(t-3, t-x)+\frac{1}{16} u(t-4, t-x)\right) ; \\
& u(m, n) \equiv \sum_{y=n}^{\left\lfloor\frac{m}{2}\right\rfloor} 2^{-(m-2 y)}\left(\begin{array}{c}
m-2 y \\
y
\end{array}\right) .
\end{aligned}
$$

The function $u(m, n)$ satisfies the following recurrence relation,

$$
u(m, n)=\frac{1}{2} u(m-1, n)+\frac{1}{2} u(m-3, n)+\theta_{m-2 x-1} 2^{2 n-m}\left(\begin{array}{c}
m-2 n \\
n-1
\end{array}\right),
$$

which implies

$$
2 u(m, n)+u(m-1, n)+u(m-2, n)= \begin{cases}2 ; & n=0, m \geq 0 \\
0 ; & n=0, m<0 \\
\sum_{y=0}^{m-2 n-1} 2^{-y}\left(\begin{array}{c}
y \\
n-1
\end{array}\right) ; & n>0 .\end{cases}
$$


Therefore, the expression (B.7) simplifies into

$$
\begin{aligned}
L_{1}(x, t)+L_{2}(x, t) & =2^{2 x} \delta_{t, x}\left(1-\delta_{t, 1}\right) \frac{3}{8}+\delta_{t, 1} \delta_{x, 1}+8 \delta_{x, 3} \delta_{t, 4}+2^{2 x} \sum_{y=0}^{2 x-t-4} 2^{-y}\left(\begin{array}{c}
y \\
t-x-1
\end{array}\right)+ \\
& +\theta_{2 x-t-3} 2^{t-1}\left(2\left(\begin{array}{c}
2 x-t-3 \\
t-x-1
\end{array}\right)+\left(\begin{array}{c}
2 x-t-3 \\
t-x-2
\end{array}\right)\right)
\end{aligned}
$$

where $\theta_{x}$ is a discrete Heaviside function,

$$
\theta_{x}= \begin{cases}1 ; & x \geq 0, \\ 0 ; & x<0 .\end{cases}
$$

The other part is obtained by observing

$$
\left\langle c, w, 0,1\left|U^{z}(1-Q) U\right| r\right\rangle=\delta_{c, 0} \delta_{w, z+1},
$$

which implies

$$
\begin{aligned}
& L_{3}(x, t)=\delta_{x, 1}+\sum_{z=\max \{1, t-2 x+1\}}^{t-x} \mathcal{A}_{x}^{0}(x, t-x-z)+\sum_{z=\max \{1, t-2 x+2\}}^{t-x} \mathcal{A}_{x}^{1}(x, t-x-z)+ \\
& +\sum_{z=\max \{1, t-2 x+3\}}^{t-x} \mathcal{A}_{x}^{1}(x-1, t-x-z) .
\end{aligned}
$$

Inserting the explicit forms of the coefficients $\mathcal{A}_{x}^{n}$ and simplifying the expression we obtain

$$
\begin{aligned}
L_{3}(x, t) & =\delta_{x, 1} \delta_{t, 1}+\theta_{2 x-t-2} 2^{t-1}\left(\begin{array}{c}
2 x-t-2 \\
t-x-1
\end{array}\right)+ \\
& +2^{x}(\underbrace{\sum_{y=0}^{x-1} 2^{y}\left(\begin{array}{c}
x-1-y \\
y
\end{array}\right)}_{\frac{2^{x}}{3}\left(1-\left(\frac{1}{2}\right)^{x}\right)}-\sum_{y=t-x}^{x-1} 2^{y}\left(\begin{array}{c}
x-1-y \\
y
\end{array}\right)) .
\end{aligned}
$$

Finally, the whole contribution of the left MPA is

$$
\begin{aligned}
L(x, t) & =3 \cdot 2^{2 x-3} \delta_{t, x}\left(1-\delta_{t, 1}\right)+2 \delta_{x, 1} \delta_{t, 1}+16 \delta_{x, 3} \delta_{t, 4}+2^{2 x} \sum_{y=0}^{2 x-t-4} 2^{-y}\left(\begin{array}{c}
y \\
t-x-1
\end{array}\right)+ \\
& +2^{2 x} \frac{1-\left(\frac{1}{2}\right)^{x}}{3}-2^{x} \sum_{y=t-x}^{x-1} 2^{y}\left(\begin{array}{c}
x-1-y \\
y
\end{array}\right)+ \\
& +\theta_{2 x-t-3} 2^{t-1}\left(3\left(\begin{array}{c}
2 x-t-3 \\
t-x-1
\end{array}\right)+2\left(\begin{array}{c}
2 x-t-3 \\
t-x-2
\end{array}\right)\right)
\end{aligned}
$$

Appendix B.2. Overlap $R(x, t)$

We start by observing

$$
V_{0}^{\prime} U^{\prime t-x}\left|r^{\prime}(t)\right\rangle=V_{0}^{\prime} U^{\prime t-x}|0, t+1,0,0\rangle=|t-x, x+1,0,0\rangle,
$$

therefore the contribution of right moving solitons to the density profile is

$$
R(x, t)=\left\langle l^{\prime}\left|\bar{T}^{x} V_{0}^{\prime} U^{\prime t-x}\right| r^{\prime}(t)\right\rangle=\left\langle l^{\prime}\left|\bar{T}^{\prime x}\right| t-x, x+1,0,0\right\rangle .
$$


We use the same approach as before; as soon as vectors $|0,0, n, 1\rangle$ are created, we compute their overlap with the left boundary vector, while we keep propagating the other vectors,

$$
\begin{aligned}
R(x, t) & =\left\langle l^{\prime}\left|\bar{T}^{\prime}(1-Q) \bar{T}^{x-1}\right| t-x, x+1,0,0\right\rangle+ \\
& +\sum_{y=1}^{x-2} 4^{x-y-1}\left\langle r\left|\bar{T}^{\prime}(1-Q) \bar{T}^{\prime y}\right| t-x, x+1,0,0\right\rangle+\left\langle r\left|\bar{T}^{\prime}\right| t-x, x+1,0,0\right\rangle
\end{aligned}
$$

where we used the fact that $\langle r|=(|r\rangle)^{T}$ is the $w=0$ part of $\left\langle l^{\prime}\right|$, i.e.

$$
\langle r|=\left\langle l^{\prime}\right|-(\langle 0,1,0,1|+\langle 0,1,2,1|)=\left\langle l^{\prime}\right| Q .
$$

The expression for $\left(1-Q^{\prime}\right) \bar{T}^{\prime y}|t-x, x+1,0,0\rangle$ is straightforwardly obtained from equation A.23 by transposing it and removing the vectors with $w=1$, therefore the right overlap reads

$$
\begin{aligned}
R(x, t) & =2 \delta_{t, 3} \delta_{x, 3}+\left(1-\delta_{t, 1}\right) \mathcal{A}_{x-1}^{0}(t-1,0) \\
& +\sum_{y=}^{x-1} 4^{x-y-1} \overline{\mathcal{A}}_{y}^{0}(t-x+y, x-y-1)+ \\
& \left.\left.+\sum_{y=}^{x-1} 2 \cdot \frac{2 x-t}{2}\right\rceil\right\} \\
y=\max \left\{1,\left\lceil\frac{2 x-t+1}{2}\right\rceil\right\} & 4^{x-y-1} \overline{\mathcal{A}}_{y-1}^{0}(t-x+y-1, x-y-1)+ \\
& +\sum_{y=\max \left\{1,\left\lceil\frac{2 x-t+2}{2}\right\rceil\right\}}^{x-y-1}\left(4 \overline{\mathcal{A}}_{y-1}^{0}(t-x+y-1, x-y)+6 \overline{\mathcal{A}}_{y-1}^{1}(t-x+y-2, x-y-1)+\right. \\
& \left.+4 \overline{\mathcal{A}}_{y-1}^{2}(t-x+y-1, x-y)+2 \overline{\mathcal{A}}_{y-1}^{2}(t-x+y-2, x-y-1)\right) .
\end{aligned}
$$

Due to the coefficients inside the sum vanishing for almost all values of $y$, the overlap can be equivalently expressed as

$$
\begin{aligned}
& R(x, t)=2 \delta_{t, 3} \delta_{x, 3}+\sum_{y=\left\lceil\frac{2 x-t-1}{2}\right\rceil}^{\infty} 2^{2 x-2 y-3} \overline{\mathcal{A}}_{y}^{0}(t-x+y, x-y-2)+ \\
& \quad+\sum_{y=\left\lceil\frac{2 x-t}{2}\right\rceil}^{\infty} 2^{2 x-2 y-3}\left(4 \overline{\mathcal{A}}_{y}^{0}(t-x+y, x-y-1)+3 \overline{\mathcal{A}}_{y}^{1}(t-x+y-1, x-y-2)+\right. \\
& \left.\quad+2 \overline{\mathcal{A}}_{y}^{2}(t-x+y, x-y-1)+\overline{\mathcal{A}}_{y}^{2}(t-x+y-1, x-y-2)\right) .
\end{aligned}
$$


Inserting the explicit values of $\overline{\mathcal{A}}_{y}^{n}$ and simplifying the whole expression yields

$$
\begin{aligned}
R(x, t) & =2 \delta_{x, 2} \delta_{t, 2}+2 \delta_{x, 3} \delta_{t, 3}+2(s(t-2)+s(t-3)+2 s(t-4)) \sum_{z=t-x}^{2 x-t-3}\left(\begin{array}{c}
2 x-t-3 \\
z
\end{array}\right)+ \\
& \left.+2\left(1-\delta_{t, 2}\right)(s(t-2)+3 s(t-4)+4 s(t-6)) \sum_{z=t-x-1}^{2 x-t-3}\left(\begin{array}{c}
2 x-t-3 \\
z
\end{array}\right) ; \quad \text { B. } 22\right) \\
s(m)= & \sum_{z=0}^{\left\lfloor\frac{m}{2}\right\rfloor} 4^{z}\left(\begin{array}{c}
m-2 z \\
z
\end{array}\right) .
\end{aligned}
$$

The function $s(m)$ satisfies the following recurrence relation,

$$
s(m+3)=s(m+2)+4 s(m),
$$

which together with the initial condition $s(0)=s(1)=s(2)=1$ implies $\S$

$s(m)+3 s(m-2)+4 s(m-4)=s(m)+2(m-1)+2 s(m-2)=2^{m}$,

and the whole contribution from the right moving solitons is

$$
R(x, t)=2 \delta_{x, 2} \delta_{t, 2}+\theta_{2 x-t-3} 2^{t-1}\left(\begin{array}{c}
2 x-t-3 \\
t-x-1
\end{array}\right)+2^{t} \sum_{z=t-x}^{2 x-t-3}\left(\begin{array}{c}
2 x-t-3 \\
z
\end{array}\right)
$$

\section{Appendix C. The free regime of the inhomogeneous quench}

In this section we present an alternative derivation of the expression for the density profile (4.7), which also provides some physical insights into the result. Before the quench, there are no solitons in the right half-infinite chain. When we join the two halfchains, the right moving solitons from the left that reach the boundary continue moving to the right unperturbed with the velocity 1 , since there are no left moving solitons to slow them down. Therefore an intermediate area with only right moving solitons is established between the vacuum and the part that contains both types of solitons. This can be seen on an example in Figure C1. Due to the maximal velocity of the solitons being $v_{\max }=1$, this area is limited to the right by $x=t$. The left border is determined by the right most possible position of the left moving solitons, which is $x=-t / 3$ due to the effective soliton speed being bounded from bellow by $1 / 3$ (see Figure C2). The ballistic part of the profile is therefore described by the $-t / 3+1 \leq x \leq t$ part of the profile in 4.7), which can also be derived by assuming that solitons enter this area randomly with uniform probability.

Let us look at the intermediate area of the chain at some fixed time $t$ and let us join two consecutive sites together, so that sites $t-(2 k-1)$ and $t-2 k$ constitute a $\S$ Note that this holds only for positive $m-4$. If $m \leq 3$, we have to explicitly express the relevant $s(m)$. 


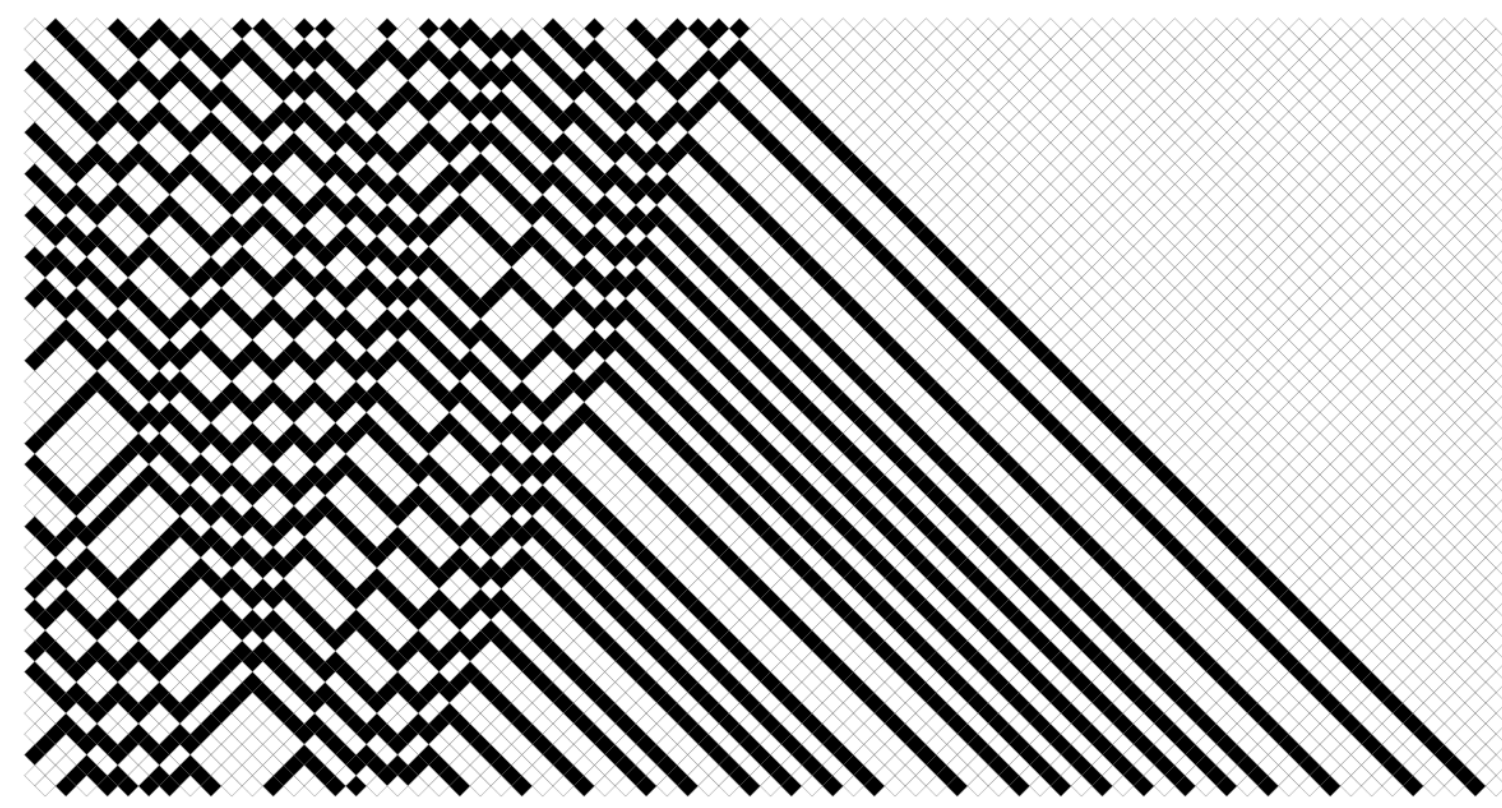

Figure C1. One realization of the inhomogeneous quench up to $t=74$. Between the empty space on the right and the area filled with left and right moving solitons, there is a section, where all the particles move to the right and do not scatter, corresponding to the free regime.

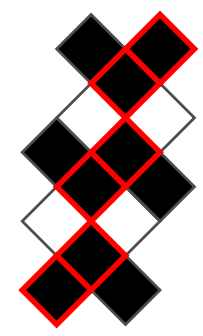

Figure C2. A path of a soliton (red bordered squares) that moves to the left with the effective velocity $1 / 3$. Due to propagation rules, the solitons can not scatter more frequently, therefore this is the slowest possible effective speed.

supersite labeled by $n=k$,

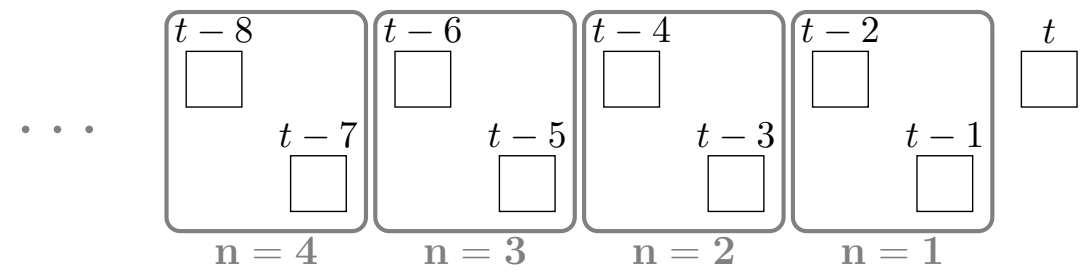

All the sites with $x \geq t$ are empty, while a site with $x \leq t-1$ is occupied if there is a right moving soliton going through it, in which case the whole supersite has to be occupied and the neighbouring supersites have to be empty. Since the solitons enter this area randomly, the site $n=1$ is occupied with probability $1 / 2$. If site $n-1$ is full, site $n$ has to be empty and if $n-1$ is empty, site $n$ is occupied with probability $1 / 2$. 
This can be expressed in a matrix form as

$$
\mathbf{x}_{n}=\left[\begin{array}{cc}
\frac{1}{2} & 1 \\
\frac{1}{2} & 0
\end{array}\right] \mathbf{x}_{n-1}=\left[\begin{array}{cc}
\frac{1}{2} & 1 \\
\frac{1}{2} & 0
\end{array}\right]^{n-1} \mathbf{x}_{1}, \quad \mathbf{x}_{n}=\left[\begin{array}{c}
1-p_{n} \\
p_{n}
\end{array}\right]
$$

where $p_{n}$ is the probability of the site $n$ being full. Taking into account $p_{1}=\frac{1}{2}$, we obtain

$$
p_{n}=\frac{1}{3}\left(1-\left(-\frac{1}{2}\right)^{n}\right)
$$

which matches the ballistic part of the profile (4.7).

\section{Appendix D. The dynamic structure factor}

As already discussed in Appendix A and Appendix B, the matrices act trivially on the vectors $\langle 0,0, n, 1|$, which implies that a general overlap, $\left\langle l(t)\left|M_{1} M_{2} \cdots M_{2 t+1}\right| r\right\rangle+$ $\left\langle l^{\prime}\left|M_{1}^{\prime} M_{2}^{\prime} \cdots M_{2 t+1}^{\prime}\right| r^{\prime}(t)\right\rangle$, can be straightforwardly determined using the projections $\langle l(t)| M_{1} M_{2} \cdots M_{j}(1-Q)$, with $j=0,1, \ldots 2 t+1$. Explicitly, the overlaps (5.5) can be expressed as

$\Delta C_{l}(x, t)=4^{t-x-1}\left\langle l(t)\left|T^{x}(1-Q) D\right| r\right\rangle+\sum_{y=0}^{t-x-2} 4^{t-x-y-2}\left\langle l(t)\left|T^{x} D \bar{T}^{y}(1-Q) \bar{T}\right| r\right\rangle$,

and

$$
\begin{aligned}
\Delta C_{r}(x, t) & =4^{t-x-1}\left\langle r\left|D^{\prime}(1-Q) T^{\prime x}\right| r^{\prime}(t)\right\rangle+\sum_{y=0}^{t-x-2} 4^{t-x-y-2}\left\langle r\left|\bar{T}^{\prime}(1-Q) \bar{T}^{\prime y} D^{\prime} T^{\prime x}\right| r^{\prime}(t)\right\rangle \\
& +\left(\left\langle l^{\prime}|-\langle r|) \bar{T}^{t-x-1} D^{\prime} T^{\prime x} \mid r^{\prime}(t)\right\rangle\right.
\end{aligned}
$$

where we introduced $D, D^{\prime}$ to denote the difference of the matrices,

$$
D=T V_{1}-V_{1} \bar{T}, \quad D^{\prime}=V_{1}^{\prime} \bar{T}^{\prime}-T^{\prime} V_{1}^{\prime} .
$$

Therefore to obtain $\Delta C_{l}(x, t)$ it suffices to express the projections $\langle l(t)| T^{x}(1-Q)$, $\langle l(t)| T^{x} D(1-Q)$ and $\langle l(t)| T^{x} D \bar{T}^{y}(1-Q)$ and then compute the relevant overlaps with the right vector $|r\rangle$ as shown in (D.1). The right moving soliton counterpart is very similar; since the matrices $W_{s}^{\prime}, V_{s}^{\prime}$ are the same as $W_{s}^{T}$ and $V_{s}^{T}$ in the $w \geq 2$ subspace, we can just take the corresponding left moving soliton vectors, transpose them, remove the terms with $w \leq 1$ (similarly as in (A.8) ) and compute the overlaps from (D.2).

The procedure is straightforward but lengthy, therefore we split it into multiple parts. In Appendix D.1 we use the relations from Appendix A to explicitly write the vectors $\langle l(t)| T^{x}(1-Q),\langle l(t)| T^{x} D(1-Q)$ and $\langle l(t)| T^{x}(1-Q) \bar{T}^{y}$ in terms of basis vectors $\langle c, w, n, a|$ by introducing the coefficients $\mathcal{A}_{x}^{n}, \mathcal{B}_{x}^{n}, \mathcal{C}_{x, y}^{n}$ and $\mathcal{D}_{x, y}^{n}$. In Appendix D.2 we proceed to express the overlaps $\Delta C_{l, r}(x, t)$. We split the overlaps into multiple parts corresponding to different coefficients and we simplify the contributions. They are expressed in terms of single binomial coefficients, their single sums and triple sums. The contributions consisting of triple sums are simplified in Appendix D.3, where also the 
whole overlaps $\Delta C_{l, r}(x, t)$ are expressed. Additionally, another subsection is included ad the end, Appendix D.4, where we show the equivalence of expressions (5.7) and (5.9) from the main text.

\section{Appendix D.1. The explicit form of different contributions to the overlaps}

We start by expressing the vectors $\langle l(t)| T^{x}(1-Q), \quad\langle l(t)| T^{x} D(1-Q)$ and $\langle l(t)| T^{x} D \bar{T}^{y}(1-Q)$. The first one can be expressed in terms of the basis vectors $\langle c, w, n, a|$ with the coefficients $\mathcal{A}_{x}^{n}(m, p)$, as introduced in (A.16) and (A.17). Acting on it with $T V_{1}-V_{1} \bar{T}$ we straightforwardly obtain

$$
\begin{aligned}
\langle l(t)| T^{x} D & (1-Q)= \\
= & 4^{x}(-2\langle x+1, t-(x+1) ; 0 ; 0|+\langle x+1, t-(x+1) ; 0 ; 1|- \\
& \quad+\langle x+1, t-(x+1) ; 0 ; 2|+\langle x+1, t-(x+1) ; 1 ; 2|)\}\langle s(x, t)| \\
& -\sum_{p} \sum_{m} \mathcal{B}_{x}^{0}(m, p)\langle x-m, t-(x+1)-p ; 1 ; 0| \\
& +\sum_{p} \sum_{m} \mathcal{B}_{x}^{1}(m, p)\langle x-m, t-(x+1)-p ; 1 ; 1| \\
& +\sum_{p} \sum_{m} \mathcal{B}_{x}^{2}(m, p)\langle x-m, t-(x+1)-p ; 1 ; 2|\langle c(x, t)|
\end{aligned}
$$

with the following coefficients

$$
\begin{aligned}
& \mathcal{B}_{x}^{0}(m, p)=2^{2 x+p-m}\left(\begin{array}{c}
m-p \\
p
\end{array}\right), \quad \mathcal{B}_{x}^{1}(m, p)=2^{2 x+p-m}\left(\begin{array}{c}
m-p-1 \\
p
\end{array}\right), \\
& \mathcal{B}_{x}^{2}(m, p)=2^{2 x+p-m-1}\left(\begin{array}{c}
m-p \\
p-1
\end{array}\right) .
\end{aligned}
$$

At this point it is convenient to split $\langle l(t)| T^{x} D \bar{T}^{y}(1-Q)$ into two parts; the first part corresponds to acting with $\bar{T}^{y}$ from right to the first two lines from (D.4),

$$
\begin{aligned}
\langle s(x, t)| \bar{T}^{y}(1-Q) & =\sum_{m, p} \mathcal{C}_{x, y}^{0}(m, p)\langle x-m, t-(x+y+1)-p ; 1 ; 0| \\
& +\sum_{m, p} \mathcal{C}_{x, y}^{1}(m, p)\langle x-m, t-(x+y+1)-p ; 1 ; 1| \\
& +\sum_{m, p} \mathcal{C}_{x, y}^{2}(m, p)\langle x-m, t-(x+y+1)-p ; 1 ; 2|
\end{aligned}
$$

where the coefficients $\mathcal{C}_{x, y}^{n}(m, p)$ are expressed in terms of $\bar{f}_{y}^{n}, \bar{g}_{y}^{n}$ and $\bar{h}_{y}^{n}$ as introduced in A.21 and A.20,

$$
\left[\begin{array}{l}
\mathcal{C}_{x, y}^{0}(m, p) \\
\mathcal{C}_{x, y}^{1}(m, p) \\
\mathcal{C}_{x, y}^{2}(m, p)
\end{array}\right]=4^{x}\left[\begin{array}{ccc}
\bar{f}_{y-1}^{0}(m, p) & \bar{f}_{y-1}^{1}(m, p) & \bar{f}_{y-1}^{2}(m, p) \\
\bar{g}_{y-1}^{0}(m, p) & \bar{g}_{y-1}^{1}(m, p) & \bar{g}_{y-1}^{2}(m, p) \\
\bar{h}_{y-1}^{0}(m, p) & \bar{h}_{y-1}^{1}(m, p) & \bar{h}_{y-1}^{2}(m, p)
\end{array}\right]\left[\begin{array}{l}
1 \\
2 \\
1
\end{array}\right]
$$


Time-dependent matrix product ansatz for interacting reversible dynamics

Similarly, the second part is

$$
\begin{aligned}
\langle c(x, t)| \bar{T}^{y}(1-Q) & =\sum_{m, p} \mathcal{D}_{x, y}^{0}(m, p)\langle x-m, t-(x+y+1)-p ; 1 ; 0| \\
& +\sum_{m, p} \mathcal{D}_{x, y}^{1}(m, p)\langle x-m, t-(x+y+1)-p ; 1 ; 1| \\
& +\sum_{m, p} \mathcal{D}_{x, y}^{2}(m, p)\langle x-m, t-(x+y+1)-p ; 1 ; 2|,
\end{aligned}
$$

with

$$
\left[\begin{array}{l}
\mathcal{D}_{x, y}^{0}(m, p) \\
\mathcal{D}_{x, y}^{1}(m, p) \\
\mathcal{D}_{x, y}^{2}(m, p)
\end{array}\right]=\sum_{c, w}\left[\begin{array}{lll}
\bar{f}_{y}^{0}(c, w) & \bar{f}_{y}^{1}(c, w) & \bar{f}_{y}^{2}(c, w) \\
\bar{g}_{y}^{0}(c, w) & \bar{g}_{y}^{1}(c, w) & \bar{g}_{y}^{2}(c, w) \\
\bar{h}_{y}^{0}(c, w) & \bar{h}_{y}^{1}(c, w) & \bar{h}_{y}^{2}(c, w)
\end{array}\right]\left[\begin{array}{c}
-\mathcal{B}_{x}^{0}(m-c, p-w) \\
\mathcal{B}_{x}^{1}(m-c, p-w) \\
\mathcal{B}_{x}^{2}(m-c, p-w)
\end{array}\right] .
$$

Appendix D.2. The explicit overlaps $\Delta C_{l, r}(x, t)$

To express the overlaps ( $(\mathrm{D} .1)$ and $(\mathrm{D} .2)$, we group the contributions from the different coefficients into separate groups,

$$
\begin{aligned}
& \Delta C_{l}(x, t) \equiv \Delta a(x, t)+\Delta b(x, t)+\Delta c(x, t)+\Delta d(x, t), \\
& \Delta C_{r}(x, t) \equiv \Delta a^{\prime}(x, t)+\Delta b^{\prime}(x, t)+\Delta c^{\prime}(x, t)+\Delta d^{\prime}(x, t),
\end{aligned}
$$

where $\Delta a(x, t)$ and $\Delta a^{\prime}(x, t)$ include all the contributions from $\mathcal{A}_{x}^{n}$ coefficients,

$$
\begin{aligned}
\Delta a(x, t) & =4^{t-x-1}\left(-\mathcal{A}_{x}^{1}(x-2, t-x-3)+\mathcal{A}_{x}^{1}(x-1, t-x-2)+\right. \\
& \left.+2 \mathcal{A}_{x}^{1}(x-1, t-x-3)-2 \mathcal{A}_{x}^{1}(x-2, t-x-2)\right) \\
\Delta a^{\prime}(x, t) & =4^{t-x-1}\left(+\mathcal{A}_{x}^{0}(x, t-x-1)+\frac{1}{2} \mathcal{A}_{x}^{0}(x, t-x-2)-\mathcal{A}_{x}^{0}(x-1, t-x-2)-\right. \\
& \left.-\mathcal{A}_{x}^{1}(x, t-x-1)+2 \mathcal{A}_{x}^{1}(x-1, t-x-1)-\frac{3}{2} \mathcal{A}_{x}^{1}(x, t-x-2)\right),
\end{aligned}
$$

$\Delta b(x, t)$ and $\Delta b^{\prime}(x, t)$ include the contributions of $\mathcal{B}_{x}^{n}$,

$$
\begin{aligned}
\Delta b(x, t) & =4^{t-x-2}\left(-4 \mathcal{B}_{x}^{0}(x, t-x-2)-\mathcal{B}_{x}^{0}(x, t-x-3)-\mathcal{B}_{x}^{0}(x-1, t-x-3)\right. \\
& +4 \mathcal{B}_{x}^{1}(x-1, t-x-2)+3 \mathcal{B}_{x}^{1}(x-1, t-x-3) \\
& \left.+2 \mathcal{B}_{x}^{2}(x, t-x-2)+2 \mathcal{B}_{x}^{2}(x-1, t-x-2)+\mathcal{B}_{x}^{2}(x-1, t-x-3)\right), \\
\Delta b^{\prime}(x, t) & =4^{t-x-2}\left(-2 \mathcal{B}_{x}^{0}(x, t-x-2)-\frac{1}{2} \mathcal{B}_{x}^{0}(x, t-x-3)+\frac{3}{2} \mathcal{B}_{x}^{1}(x-1, t-x-3)+\right. \\
& \left.+\mathcal{B}_{x}^{2}(x, t-x-2)+\frac{1}{2} \mathcal{B}_{x}^{2}(x-1, t-x-3)\right)
\end{aligned}
$$


Time-dependent matrix product ansatz for interacting reversible dynamics

$\Delta c(x, t)$ and $\Delta c^{\prime}(x, t)$ contain the contributions from $\mathcal{C}_{x, y}^{n}$,

$$
\begin{aligned}
\Delta c(x, t) & =\sum_{y=1}^{t-x-2} 4^{t-x-y-2}\left(4 \mathcal{C}_{x, y}^{0}(x, t-x-y-2)+\mathcal{C}_{x, y}^{0}(x, t-x-y-3)+\right. \\
& +\mathcal{C}_{x, y}^{0}(x-1, t-x-y-3)+4 \mathcal{C}_{x, y}^{1}(x-1, t-x-y-2)+ \\
& +3 \mathcal{C}_{x, y}^{1}(x-1, t-x-y-3)+2 \mathcal{C}_{x, y}^{1}(x, t-x-y-2)+ \\
& \left.+2 \mathcal{C}_{x, y}^{2}(x-1, t-x-y-2)+\mathcal{C}_{x, y}^{2}(x-1, t-x-y-3)\right) \\
\Delta c^{\prime}(x, t) & =\sum_{y=1}^{t-x-2} 4^{t-x-y-2}\left(2 \mathcal{C}_{x, y}^{0}(x, t-x-y-2)+\frac{1}{2} \mathcal{C}_{x, y}^{0}(x, t-x-y-3)+\right. \\
& +\frac{3}{2} \mathcal{C}_{x, y}^{1}(x-1, t-x-y-3)+\mathcal{C}_{x, y}^{2}(x, t-x-y-2)+ \\
& \left.+\frac{1}{2} \mathcal{C}_{x, y}^{2}(x-1, t-x-y-3)\right)
\end{aligned}
$$

and $\Delta d(x, t), \Delta d^{\prime}(x, t)$ contain the contributions from $\mathcal{D}_{x, y}^{n}$,

$$
\begin{aligned}
\Delta d(x, t) & =\sum_{y=1}^{t-x-2} 4^{t-x-y-2}\left(4 \mathcal{D}_{x, y}^{0}(x, t-x-y-2)+\mathcal{D}_{x, y}^{0}(x, t-x-y-3)+\right. \\
& +\mathcal{D}_{x, y}^{0}(x-1, t-x-y-3)+4 \mathcal{D}_{x, y}^{1}(x-1, t-x-y-2)+ \\
& +3 \mathcal{D}_{x, y}^{1}(x-1, t-x-y-3)+2 \mathcal{D}_{x, y}^{2}(x, t-x-y-2)+ \\
& \left.+2 \mathcal{D}_{x, y}^{2}(x-1, t-x-y-2)+\mathcal{D}_{x, y}^{2}(x-1, t-x-y-3)\right) \\
\Delta d^{\prime}(x, t) & =\sum_{y=1}^{t-x-2} 4^{t-x-y-2}\left(2 \mathcal{D}_{x, y}^{0}(x, t-x-y-2)+\frac{1}{2} \mathcal{D}_{x, y}^{0}(x, t-x-y-3)+\right. \\
& +\frac{3}{2} \mathcal{D}_{x, y}^{1}(x-1, t-x-y-3)+\mathcal{D}_{x, y}^{2}(x, t-x-y-2)+ \\
& \left.+\frac{1}{2} \mathcal{D}_{x, y}^{2}(x-1, t-x-y-3)\right) .
\end{aligned}
$$

The contributions $\Delta a(x, t), \Delta b(x, t), \Delta a^{\prime}(x, t)$ and $\Delta b^{\prime}(x, t)$ can be expressed in terms of simple binomial coefficients by plugging the coefficients $\mathcal{A}_{x}^{n}$, $\mathcal{B}_{x}^{n}$ into equations (D.11) and (D.12),

$$
\begin{aligned}
\Delta a(x, t)+\Delta b(x, t) & =\theta_{2 x-t-2} 2^{3 t-2 x-3}\left(\left(\begin{array}{c}
2 x-t-2 \\
t-x-3
\end{array}\right)-\left(\begin{array}{c}
2 x-t-2 \\
t-x-2
\end{array}\right)\right)- \\
& -\theta_{2 x-t+1} 2^{3 t-2 x-7}\left(\left(\begin{array}{c}
2 x-t+1 \\
t-x-4
\end{array}\right)-\left(\begin{array}{c}
2 x-t+1 \\
t-x-3
\end{array}\right)\right) \\
\Delta a^{\prime}(x, t)+\Delta b^{\prime}(x, t) & =\theta_{2 x-t-2}\left(2\left(\begin{array}{c}
2 x-t-2 \\
t-x-1
\end{array}\right)-\left(\begin{array}{c}
2 x-t-1 \\
t-x-3
\end{array}\right)\right)- \\
& -\theta_{2 x-t+1} 2^{3 t-2 x-8}\left(\left(\begin{array}{c}
2 x-t+1 \\
t-x-3
\end{array}\right)-\left(\begin{array}{c}
2 x-t+1 \\
t-x-4
\end{array}\right)\right) .
\end{aligned}
$$


Time-dependent matrix product ansatz for interacting reversible dynamics

Similarly, the sums (D.13) can be simplified into the following form,

$\Delta c(x, t)=\theta_{t-2 x-4} 2^{t+2 x-1}\left(6\left(\begin{array}{c}t-2 x-4 \\ x\end{array}\right)+5\left(\begin{array}{c}t-2 x-4 \\ x-1\end{array}\right)+\left(\begin{array}{c}t-2 x-4 \\ x-2\end{array}\right)\right)$,

$\Delta c(x, t)^{\prime}=\theta_{t-2 x-4} 2^{t+2 x-1}\left(3\left(\begin{array}{c}t-2 x-4 \\ x\end{array}\right)+\left(\begin{array}{c}t-2 x-4 \\ x-1\end{array}\right)\right)$,

by observing that for any $u \geq 0$ the following holds,

$\sum_{m=0}^{\left\lfloor\frac{u}{2}\right\rfloor} 4^{m}\left(\begin{array}{c}u-2 m \\ m\end{array}\right)=2^{u-1}+\frac{1-\frac{\mathrm{i}}{\sqrt{7}}}{4}\left(\frac{-1+\mathrm{i} \sqrt{7}}{2}\right)^{u}+\frac{1+\frac{\mathrm{i}}{\sqrt{7}}}{4}\left(\frac{-1-\mathrm{i} \sqrt{7}}{2}\right)^{u} \equiv a_{u}$.

However, simplifying the contributions $\Delta d(x, t)$ and $\Delta d^{\prime}(x, t)$ requires a bit more work.

Appendix D.3. Contributions $\Delta d(x, t)$ and $\Delta d^{\prime}(x, t)$

We start by noting that both the remaining contributions can be expressed in terms of the following triple sum,

$$
\begin{aligned}
& s_{x, t}(\alpha, \beta, \gamma)=\sum_{z=0}^{\min \{x+\alpha, t-x+\beta\}} \sum_{y=0}^{\min \{x+\alpha, t-x+\beta\}-z} 2^{-(x+\alpha-y-z)}\left(\begin{array}{c}
x+\alpha-y-z \\
y
\end{array}\right) \times \\
& \times 2^{-(t-x+\beta-y-z)}\left(\begin{array}{c}
t-x+\beta-y-z \\
z
\end{array}\right) \sum_{w=0}^{\frac{1}{2}(t-x+\gamma-y+z)} 2^{-(t-x+\gamma-y+z-2 w)}\left(\begin{array}{c}
t-x+\gamma-y+x-2 w \\
w
\end{array}\right),
\end{aligned}
$$

as

$$
\begin{aligned}
\Delta d(x, t) & -2 \Delta d^{\prime}(x, t)=2^{3 t-2 x-6}\left(\begin{array}{c}
2 x-t+2 \\
t-x-3
\end{array}\right)+ \\
& +2^{2 t-5}\left(s_{x, t}(-3,-4,-3)+s_{x, t}(-3,-4,-2)+2 s_{x, t}(-3,-4,-1)+\right. \\
& +s_{x, t}(-2,-3,-6)+s_{x, t}(-2,-3,-5)+2 s_{x, t}(-2,-3,-4)- \\
& \left.-2 s_{x, t}(-1,-3,-4)-2 s_{x, t}(-1,-3,-3)-4 s_{x, t}(-1,-3,-2)\right)
\end{aligned}
$$

and

$$
\begin{aligned}
\Delta d^{\prime}(x, t) & =2^{3 t-2 x-8}\left(8\left(\begin{array}{c}
2 x-t+2 \\
t-x-2
\end{array}\right)+\left(\begin{array}{c}
2 x-t+3 \\
t-x-3
\end{array}\right)\right)+ \\
& +2^{2 t-7}\left(s_{x, t}(-3,-5,-4)+3 s_{x, t}(-3,-5,-2)+s_{x, t}(-2,-4,-7)+\right. \\
& +3 s_{x, t}(-2,-4,-5)+2 s_{x, t}(-2,-4,-3)+2 s_{x, t}(-2,-4,-2)+ \\
& +8 s_{x, t}(-2,-3,-1)-2 s_{x, t}(-1,-4,-5)-6 s_{x, t}(-1,-4,-3)+ \\
& +2 s_{x, t}(-1,-3,-6)+2 s_{x, t}(-1,-3,-5)+8 s_{x, t}(-1,-2,-4)- \\
& \left.-4 s_{x, t}(0,-3,-4)-4 s_{x, t}(0,-3,-3)-16 s_{x, t}(0,-2,-2)\right) .
\end{aligned}
$$

Note that instead of explicitly expressing $\Delta d(x, t)$ we simplified the expressions a bit by introducing $\Delta d(x, t)-2 \Delta d^{\prime}(x, t)$. 
We first observe that the inner-most sum in (D.18) can be evaluated,

$$
\sum_{w=0}^{\frac{u}{2}} 2^{-u+2 w}\left(\begin{array}{c}
u-2 w \\
w
\end{array}\right)=2^{-u} a_{u}
$$

with $a_{u}$ defined in (D.17). If $u \geq 0$, the coefficients $a_{u}$ satisfy the following recurrence relation,

$$
a_{u+1}=a_{u}+4 a_{u-2} .
$$

This enables us to rewrite the expression (D.19) in terms of simpler double sums,

$$
\bar{s}(m, n)=\sum_{z=0}^{\min \{m, n\}} \sum_{y=0}^{\min \{m, n\}-z} 4^{y+z}\left(\begin{array}{c}
m-y-z \\
y
\end{array}\right)\left(\begin{array}{c}
n-y-z \\
z
\end{array}\right),
$$

by grouping together the terms $s_{x, t}(\alpha, \beta, \gamma)$ with the same $\alpha, \beta$. Explicitly,

$s_{x, t}(\alpha, \beta, \gamma)+s_{x, t}(\alpha, \beta, \gamma+1)+2 s_{x, t}(\alpha, \beta, \gamma+2) \mapsto 2^{-(t+\alpha+\beta)} \bar{s}(x+\alpha, t-x+\beta)$.

Furthermore, we have to subtract the terms that contain $a_{n}$ with $n<0$, since the relation does not hold for them. Taking care of these corner cases, the contribution $\Delta d(x, t)-2 \Delta d^{\prime}(x, t)$ can be rewritten as

$$
\begin{aligned}
& \Delta d(x, t)-2 \Delta d^{\prime}(x, t)=2^{3 t-2 x-6}\left(\begin{array}{c}
2 x-t+1 \\
t-x-3
\end{array}\right)-2^{3 t-2 x-5}\left(\begin{array}{c}
2 x-t+1 \\
t-x-3
\end{array}\right)+ \\
& +2^{t+3} \bar{s}(x-3, t-x-4)+2^{t+1} \bar{s}(x-2, t-x-3)-2^{t+1} \bar{s}(x-1, t-x-3) .
\end{aligned}
$$

It is possible to further simplify this result by noting that the functions $\bar{s}(m, n)$ satisfy the following two relations,

$$
\begin{aligned}
& \bar{s}(m+1, n)=\bar{s}(m, n)+4 \bar{s}(m-1, n-1)+\varepsilon(m, n), \\
& \bar{s}(m, n+1)=\bar{s}(m, n)+4 \bar{s}(m-1, n-1)+\eta(m, n), \\
& \varepsilon(m, n)=\theta_{n-m-1} 4^{m+1}\left(\begin{array}{c}
n-m-1 \\
m+1
\end{array}\right), \quad \eta(m, n)=\theta_{m-n-1} 4^{n+1}\left(\begin{array}{c}
m-n-1 \\
n+1
\end{array}\right),
\end{aligned}
$$

which enable us to obtain

$$
\Delta d(x, t)-2 \Delta d^{\prime}(x, t)= \begin{cases}-2^{2 x+t-1}\left(\begin{array}{c}
t-2 x-2 \\
x-1
\end{array}\right) ; & x \leq \frac{t-2}{2}, \\
-2^{3 t-2 x-6}\left(\left(\begin{array}{c}
2 x-t+1 \\
t-x-3
\end{array}\right)-\left(\begin{array}{c}
2 x-t+1 \\
t-x-4
\end{array}\right)\right) ; & x \geq \frac{t-1}{2} .\end{cases}
$$

The contribution (D.20) is a bit more complicated, since the sums $s_{x, t}(\alpha, \beta, \gamma)$ with the same $\alpha, \beta$ do not simplify as before. Therefore we split the coefficients $a_{n}$ into two parts,

$$
2^{-n} a_{n}=\frac{1}{2}+b_{n}
$$

and we treat the different contributions to $\Delta d^{\prime}(x, t)$ separately,

$$
\Delta d^{\prime}(x, t)=\Delta d_{c}^{\prime}(x, t)+\Delta d_{r}^{\prime}(x, t)+\Delta d_{i}^{\prime}(x, t),
$$

where $\Delta d_{c}^{\prime}(x, t)$ includes all the constant terms,

$$
\Delta d_{c}^{\prime}(x, t)=2^{3 t-2 x-8}\left(8\left(\begin{array}{c}
2 x-t+2 \\
t-x-2
\end{array}\right)-\left(\begin{array}{c}
2 x-t+2 \\
t-x-3
\end{array}\right)\right),
$$


Time-dependent matrix product ansatz for interacting reversible dynamics

and $\Delta d_{i, r}^{\prime}(x, t)$ correspond to different parts of the coefficients $a_{n}$. Explicitly,

$$
\begin{aligned}
\Delta d_{r}^{\prime}(x, t) & =2^{t-2}(16 \bar{s}(x-3, t-x-5)+8 \bar{s}(x-2, t-x-4)+4 \bar{s}(x-2, t-x-3)- \\
& -4 \bar{s}(x-1, t-x-4)+\bar{s}(x-1, t-x-3)+\bar{s}(x-1, t-x-2)- \\
& -\bar{s}(x, t-x-3)-\bar{s}(x, t-x-2))
\end{aligned}
$$

and

$$
\begin{aligned}
\Delta d_{i}^{\prime}(x, t) & =-2^{t-1}\left(4 \bar{s}_{2}(x-3, t-x-5)-4 \bar{s}_{3}(x-3, t-x-5)+\right. \\
& +2 \bar{s}_{1}(x-2, t-x-4)-2 \bar{s}_{2}(x-2, t-x-4)-4 \bar{s}_{2}(x-2, t-x-3)- \\
& -\bar{s}_{0}(x-1, t-x-4)+\bar{s}_{1}(x-1, t-x-4)-\bar{s}_{0}(x-1, t-x-3)- \\
& -2 \bar{s}_{1}(x-1, t-x-3)+\bar{s}_{0}(x-1, t-x-2)-2 \bar{s}_{1}(x-1, t-x-2)- \\
& \left.-\bar{s}_{1}(x, t-x-3)+\bar{s}_{0}(x, t-x-2)\right)
\end{aligned}
$$

with the generalized sums $\bar{s}_{\gamma}(m, n)$ defined as

$$
\bar{s}_{\gamma}(m, n)=\sum_{z=0}^{\min \{m, n\}} \sum_{y=0}^{\min \{m, n\}-z} 4^{y+z}\left(\begin{array}{c}
m-y-z \\
y
\end{array}\right)\left(\begin{array}{c}
n-y-z \\
z
\end{array}\right) b_{n+1+\gamma-y-z} .
$$

Similarly as before, the contribution (D.31) reduces into

$$
\begin{aligned}
\Delta d_{r}^{\prime}(x, t) & =2^{t-2}(-4 \eta(x-2, t-x-4)-\eta(x-1, t-x-3)+\eta(x, t-x-3)- \\
& -2 \varepsilon(x-1, t-x-2))= \\
& =-\theta_{t-2 x-2} 2^{2 x+t-1}\left(\begin{array}{c}
t-2 x-2 \\
x
\end{array}\right) .
\end{aligned}
$$

In order to simplify the last part (D.32), we first observe that the following relations hold,

$$
\begin{aligned}
& \bar{s}_{\gamma}(m+1, n)=\bar{s}_{\gamma}(m, n)+4 \bar{s}_{\gamma}(m-1, n-1)+\varepsilon_{\gamma}(m, n), \\
& \bar{s}_{\gamma}(m, n+1)=\bar{s}_{\gamma+1}(m, n)+4 \bar{s}_{\gamma+3}(m-1, n-1)+\eta_{\gamma}(m, n), \\
& \varepsilon_{\gamma}(m, n)=\theta_{n-m-1} 4^{m+1}\left(\begin{array}{c}
n-(m+1) \\
m+1
\end{array}\right) b_{n+m+1+\gamma}, \\
& \eta_{\gamma}(m, n)=\theta_{m-n-1} 4^{n+1}\left(\begin{array}{c}
m-(n+1) \\
n+1
\end{array}\right) b_{\gamma} .
\end{aligned}
$$

Using them, we obtain,

$$
\begin{aligned}
\Delta d_{i}^{\prime}(x, t) & =-2^{t-1}\left(\eta_{0}(x, t-x-3)-\eta_{1}(x-1, t-x-3)-4 \eta_{2}(x-2, t-x-4)+\right. \\
& \left.+4 \eta_{3}(x-1, t-x-3)\right)= \\
& =-\theta_{2 x-t+1} 2^{3 t-2 x-7}\left(3\left(\begin{array}{c}
2 x-t+1 \\
t-x-3
\end{array}\right)+4\left(\begin{array}{c}
2 x-t+1 \\
t-x-2
\end{array}\right)\right)
\end{aligned}
$$


which yields

$$
\Delta d^{\prime}(x, t)= \begin{cases}-2^{2 x+t-1}\left(\begin{array}{c}
t-2 x-2 \\
x
\end{array}\right) ; & x \leq \frac{t-2}{2} \\
2^{3 t-2 x-9}\left(\left(\begin{array}{c}
2 x-t+1 \\
t-x-3
\end{array}\right)-\left(\begin{array}{c}
2 x-t+1 \\
t-x-4
\end{array}\right)\right) ; & x \geq \frac{t-1}{2} .\end{cases}
$$

By combining the equations (D.15), (D.16), (D.27) and (D.37), we can finally express the left and right overlap as

$$
\begin{aligned}
& \Delta C_{l}(x, t)= \begin{cases}2^{2 x+t-1}\left(4\left(\begin{array}{c}
t-2 x-4 \\
x
\end{array}\right)-3\left(\begin{array}{c}
t-2 x-4 \\
x-2
\end{array}\right)-\left(\begin{array}{c}
t-2 x-4 \\
x-3
\end{array}\right)\right), & x \leq \frac{t-4}{2}, \\
0, & \frac{t-3}{2} \leq x \leq \frac{t+1}{2}, \\
2^{3 t-2 x-3}\left(\left(\begin{array}{c}
2 x-t-2 \\
t-x-3
\end{array}\right)-\left(\begin{array}{c}
2 x-t-2 \\
t-x-2
\end{array}\right)\right), & \frac{t+2}{2} \leq x,\end{cases} \\
& \Delta C_{r}(x, t)= \begin{cases}2^{2 x+t-1}\left(2\left(\begin{array}{c}
t-2 x-4 \\
x
\end{array}\right)-\left(\begin{array}{c}
t-2 x-4 \\
x-1
\end{array}\right)-\left(\begin{array}{c}
t-2 x-4 \\
x-2
\end{array}\right)\right), & x \leq \frac{t-4}{2}, \\
0, & \frac{t-3}{2} \leq x \leq \frac{t+1}{2}, \\
2^{3 t-2 x-3}\left(2\left(\begin{array}{c}
2 x-t-2 \\
t-x-1
\end{array}\right)-\left(\begin{array}{c}
2 x-t-2 \\
t-x-3
\end{array}\right)-\left(\begin{array}{c}
2 x-t-2 \\
t-x-4
\end{array}\right)\right), & \frac{t+2}{2} \leq x .\end{cases}
\end{aligned}
$$

Appendix D.4. The equation (5.9)

To show that (5.9) is equivalent to (5.7), it suffices to prove that the polynomial $\tilde{p}(u, x)$, defined as

$$
\tilde{p}(u, x)=\sum_{n=2 x}^{3 x}\left(\prod_{\substack{j=2 x \\ j \neq n}}^{3 x} \frac{u-j}{n-j}\right)(2 s(n)-s(n+1)),
$$

is equivalent to the sum $p(u, x)$,

$$
p(u, x)=\sum_{m=0}^{x} 4^{m}\left(2\left(\begin{array}{c}
u-2 m \\
m
\end{array}\right)-\left(\begin{array}{c}
u+1-2 m \\
m
\end{array}\right)\right),
$$

where $s(u)=p\left(u,\left\lfloor\frac{u}{2}\right\rfloor\right)$ was defined in the main text $\|$ and $u \geq 0,2 x \leq u$. Clearly, if $\frac{u}{2} \geq x \geq \frac{u+1}{3}$, both expressions coincide, therefore it is sufficient to show that $\tilde{p}(u, x)$ satisfies the same relation as $p(u, x)$,

$p(u, x)=p(u-1, x)+4 p(u-2, x)-4^{x+1}\left(2\left(\begin{array}{c}u-2 x-3 \\ x\end{array}\right)-\left(\begin{array}{c}u-2 x-2 \\ x\end{array}\right)\right)$.

$\|$ This is also the same as $a_{u}$, defined in (D.17). 
After some straightforward manipulation of the sums, we obtain the following $\tilde{p}(u, x)-\tilde{p}(u-1, x)-4 \tilde{p}(u-3, x)=$ $=\sum_{n=2 x}^{3 x}\left(\prod_{\substack{j=2 x \\ j \neq n}}^{3 x} \frac{u-j}{n-j}\right) \overbrace{(-s(n+1)+3 s(n)-2 s(n-1)+4 s(n-2)-8 s(n-3))}^{=0}+$

$+\sum_{n=2 x+2}^{3 x+3}(-1)^{n+3 x}\left(\begin{array}{c}x+1 \\ n-2 x-2\end{array}\right)\left(\begin{array}{c}u-2 x-3 \\ x\end{array}\right)(8 s(n-3)-4 s(n-2))+$

$+\sum_{n=2 x+1}^{3 x+2}(-1)^{n+3 x}\left(\begin{array}{c}x+1 \\ n-2 x-1\end{array}\right)\left(\begin{array}{c}u-2 x-2 \\ x\end{array}\right)(8 s(n-3)-4 s(n-2))+$

$+\sum_{n=2 x}^{3 x+1}(-1)^{n+3 x}\left(\begin{array}{c}x+1 \\ n-2 x\end{array}\right)\left(\begin{array}{c}u-2 x-1 \\ x\end{array}\right) \overbrace{(-s(n)+2 s(n-1)-4 s(n-2)+8 s(n-3))}^{=2 s(n)-s(n+1)}$.

Expressing it in terms of the sums $r(x, \alpha)=\sum_{n=0}^{x+1}(-1)^{n}\left(\begin{array}{c}x+1 \\ n\end{array}\right) s(n+\alpha)$ and taking into account the following properties,

$$
\left.\begin{array}{l}
r(x+1, \alpha)=-4 r(x, \alpha-2) \\
r(0, \alpha)=-4 s(\alpha-2)
\end{array}\right\} r(x, \alpha)=(-4)^{x+1} s(\alpha-2 x-2),
$$

yields

$\tilde{p}(u, x)-\tilde{p}(u-1, x)-4 \tilde{p}(u-2, x)=-4^{x+1}\left(2\left(\begin{array}{c}u-2 x-3 \\ x\end{array}\right)-\left(\begin{array}{c}u-2 x-2 \\ x\end{array}\right)\right)$. 